\title{
High-resolution characterization of TiN diffusion barrier layers
}

\author{
Marlene Mühlbacher
}

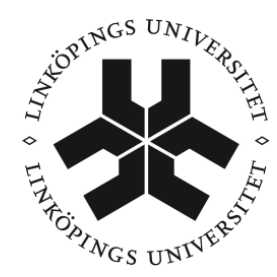

\section{Linköping University INSTITUTE OF TECHNOLOGY}

Thin Film Physics Division

Department of Physics, Chemistry, and Biology (IFM) Linköping University, SE-581 83 Linköping, Sweden 
High-resolution characterization of TiN diffusion barrier layers

(C) Marlene Mühlbacher, 2015

Except for Paper I ( Elsevier B.V., reprinted with permission

Printed in Sweden by LiU-Tryck, Linköping, Sweden, 2015

ISBN 978-91-7685-994-0

ISSN 0280-7971 
What we become depends on what we read after all of the professors have finished with us. The greatest university of all is a collection of books.

- Thomas Carlyle

Would it save you a lot of time if I just gave up and went mad now?

- Douglas Adams, The Hitchhiker's Guide to the Galaxy 



\section{ACKNOWLEDGMENTS}

I am deeply grateful to my supervisors Lars Hultman and Christian Mitterer for giving me the chance to work in two wonderful groups where I could not only expand my knowledge about thin film physics but also got the opportunity to broaden my horizon by interacting with amazing people from all over the world.

An equally big thank you is due to my co-supervisors Jun Lu and Nina Schalk, who always took the time to listen, discuss and show the path forward.

I would like to thank all the technical and administrative staff for paving the way through challenging technical and bureaucratic matters, most of all Thomas Lingefelt, the "TEM whisperer", and Bernhard Sartory, the "FIB wizard".

I am also grateful to all of my co-authors. It was a pleasure to do research with you!

Many thanks go to my colleagues in Linköping and Leoben for the pleasant and stimulating work environment.

An important part of my time in Linköping was spent with all the new friends I found here: Susann, Anke, Joseph, Agne, Jonas, Daniel, Joanna, György, Tuomas, Iris - I would like to thank you for the laughs we shared and for making me feel at home (and, where applicable, for sharing apple beers).

I would also like to thank all my friends in Leoben for always welcoming me back, especially July, who managed to visit (almost) every year.

Last but not least, I would like to thank my parents for their financial support, and, most importantly, for taking good care and sending many pictures of my dog and cats. 



\section{ABSTRACT}

Titanium nitride (TiN) films are widely applied as diffusion barrier layers in microelectronic devices. The continued miniaturization of such devices not only poses new challenges to material systems design, but also puts high demands on characterization techniques. To gain understanding of diffusion processes that can eventually lead to failure of the barrier layer and thus of the whole device, it is essential to develop routines to chemically and structurally investigate these layers down to the atomic scale. In the present study, model TiN diffusion barriers with a $\mathrm{Cu}$ overlayer acting as the diffusion source were grown by reactive magnetron sputtering on $\mathrm{MgO}(001)$ and thermally oxidized $\mathrm{Si}(001)$ substrates. Cross-sectional transmission electron microscopy (XTEM) of the pristine samples revealed epitaxial, single-crystalline growth of $\mathrm{TiN}$ on $\mathrm{MgO}(001)$, while the polycrystalline TiN grown on $\mathrm{Si}(001)$ exhibited a [001]-oriented columnar microstructure. Various annealing treatments were carried out to induce diffusion of $\mathrm{Cu}$ into the TiN layer. Subsequently, XTEM images were recorded with a highangle annular dark field detector, which provides strong elemental contrast, to illuminate the correlation between the structure and the barrier efficiency of the single- and polycrystalline TiN layers. Particular regions of interest were investigated more closely by energy dispersive X-ray (EDX) mapping. These investigations are completed by atom probe tomography (APT) studies, which provide a three-dimensional insight into the elemental distribution at the nearinterface region with atomic chemical resolution and high sensitivity. In case of the single-crystalline barrier, a uniform $\mathrm{Cu}$-enriched diffusion layer of $12 \mathrm{~nm}$ could be detected at the interface after an annealing treatment at $1000{ }^{\circ} \mathrm{C}$ for $12 \mathrm{~h}$. This excellent barrier performance can be attributed to the lack of fast diffusion paths such as grain boundaries. Moreover, density-functional theory calculations predict a stoichiometry-dependent atomic diffusion mechanism of $\mathrm{Cu}$ in bulk TiN, with $\mathrm{Cu}$ diffusing on the $\mathrm{N}$-sublattice for the experimental $\mathrm{N} / \mathrm{Ti}$ ratio. In comparison, the polycrystalline TiN layers exhibited grain boundaries reaching from the $\mathrm{Cu}$-TiN interface to the substrate, thus providing direct diffusion paths for $\mathrm{Cu}$. However, the microstructure of these columnar layers was still dense without open porosity or voids, so that the onset of grain boundary diffusion could only be found after annealing at $900{ }^{\circ} \mathrm{C}$ for $1 \mathrm{~h}$.

The present study shows how to combine two high resolution state-of-the-art methods, TEM and APT, to characterize model TiN diffusion barriers. It is shown how to correlate the microstructure with the performance of the barrier layer by two-dimensional EDX mapping and three-dimensional APT. Highly effective $\mathrm{Cu}$-diffusion barrier function is thus demonstrated for single-crystal $\operatorname{TiN}(001)$ (up to $1000^{\circ} \mathrm{C}$ ) and dense polycrystalline $\operatorname{TiN}\left(900^{\circ} \mathrm{C}\right.$ ). 



\section{INCLUDED PUBLICATIONS}

Paper I. Copper diffusion into single-crystalline TiN studied by transmission electron microscopy and atom probe tomography.

M. Mühlbacher, F. Mendez-Martin, B. Sartory, N. Schalk, J. Keckes, J. Lu, L. Hultman, and C. Mitterer.

Thin Solid Films. 574 (2015) 103-109.

Paper II. Cu diffusion in single-crystal and polycrystalline TiN barrier layers: A high-resolution experimental study supported by first-principles calculations.

M. Mühlbacher, A. S. Bochkarev, F. Mendez-Martin, B. Sartory, L. Chitu, M. N. Popov, P. Puschnig, J. Spitaler, H. Ding, N. Schalk, J. Lu, L. Hultman, and C. Mitterer.

Submitted for publication. 



\section{PREFACE}

This thesis summarizes my research work conducted in equal parts at the Department of Physics, Chemistry and Biology (IFM) within the Thin Film Physics Division at Linköping University (Sweden) and at the Chair of Functional Materials and Materials Systems at Montanuniversität Leoben (Austria) from September 2012 to August 2015.

The present study demonstrates how to combine two high-resolution state-of-theart methods, transmission electron microscopy and atom probe tomography, to characterize the interdiffusion mechanisms in model TiN/Cu stacks down to the atomic scale. Experimental findings are complemented by numerical simulations to provide a comprehensive description of diffusion phenomena in materials relevant for microelectronics. The main results are presented in the appended papers.

Financial support by the Austrian Federal Government (in particular from Bundesministerium für Verkehr, Innovation und Technologie and Bundesministerium für Wirtschaft, Familie und Jugend) represented by Österreichische Forschungsförderungsgesellschaft $\mathrm{mbH}$ and the Styrian and the Tyrolean Provincial Government, represented by Steirische Wirtschaftsförderungsgesellschaft $\mathrm{mbH}$ and Standortagentur Tirol, within the framework of the COMET Funding Programme is gratefully acknowledged.

The Ultra Electron Microscopy Laboratory at Linköping University operated by the Thin Film Physics Division is supported by the Swedish Knut and Alice Wallenberg Foundation. 



\section{CONTENTS}

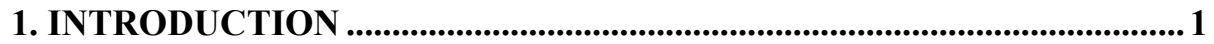

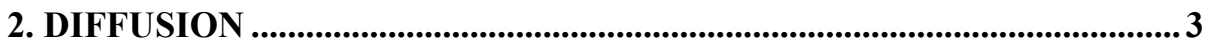

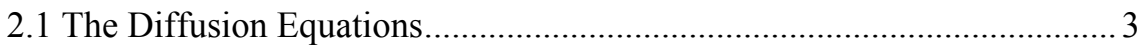

2.2 Solution of the Diffusion Equations for Thin Films.................................. 5

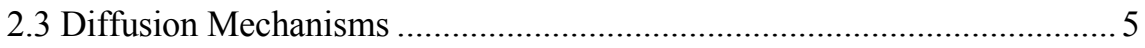

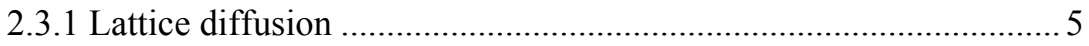

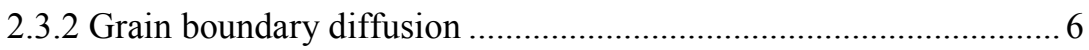

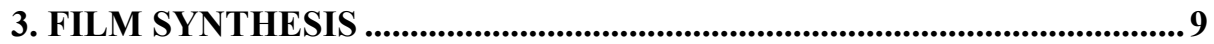

3.1 Magnetron Sputter Deposition ......................................................... 9

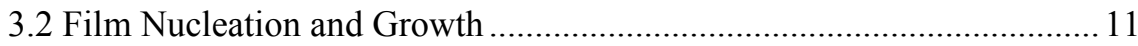

3.2.1 Nucleation and early stages of growth .......................................... 11

3.2.2 Epitaxial growth of single-crystalline films .................................. 13

3.2.3 Polycrystalline films ............................................................... 15

3.2.4 Effects of ion irradiation............................................................... 16

4. FUNCTIONAL TITANIUM NITRIDE FILMS …….................................. 19

4.1 Structure and Properties of TiN............................................................ 19

4.1.1 Composition, structure and mechanical properties ........................ 19

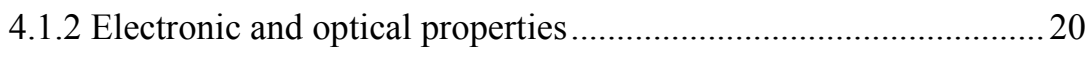

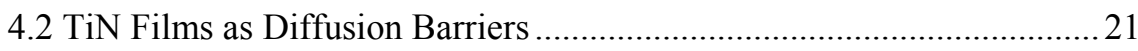

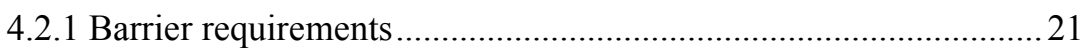

4.2.2 Developments in TiN diffusion barrier design............................... 22

5. TRANSMISSION ELECTRON MICROSCOPY ….....................................25

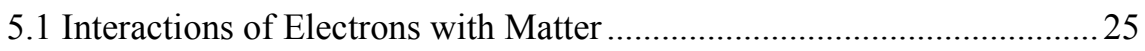

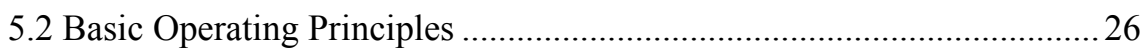

5.2.1 Bright-field and dark-field imaging .............................................. 28

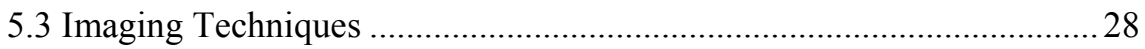

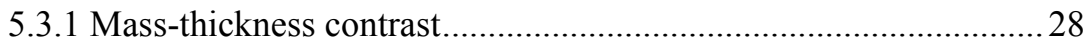

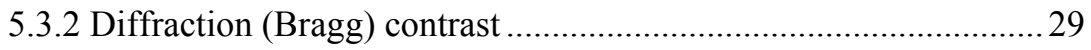

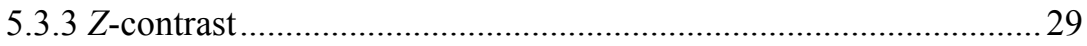

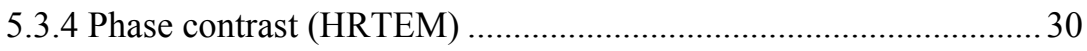

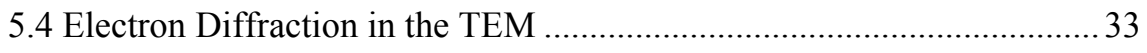

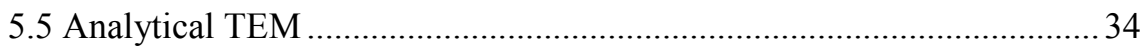


6. ATOM PROBE TOMOGRAPHY ..........................................................3 37

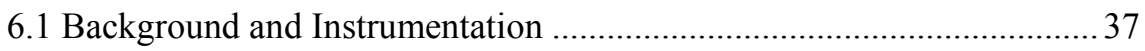

6.1.1 From field ion microscopy to atom probe tomography ................ 37

6.1.2 Operating principle................................................................. 38

6.2 Specimen Preparation ................................................................. 40

6.2.1 Site-specific FIB sample preparation ...................................... 40

6.3 Data Collection and Reconstruction ................................................ 42

6.3.1 Measurement parameters ..................................................... 42

6.3.2 Data processing and 3D reconstruction.................................. 42

6.4 Application to Diffusion Studies .................................................... 46

6.4.1 Chemical depth profiling .................................................... 46

6.4.2 Isoconcentration surfaces ........................................................ 47

7. CONCLUSIONS AND OUTLOOK ..........................................................49

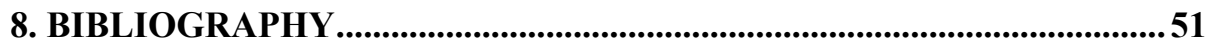

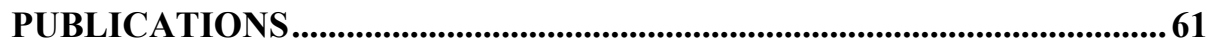




\section{INTRODUCTION}

No other technology has impacted everyday life in the late $20^{\text {th }}$ and early $21^{\text {st }}$ century like the advent and developments in microelectronics. From the International Space Station to commercial airplanes, from dishwashers to mobile phones - integrated circuits (ICs), perhaps better known as microchips, have been an essential part of virtually all recent technological advances. Still, the drive for smaller, faster, more reliable and less expensive ICs with more diverse functionality has not let up. A common measure of progress in IC design is the degree of device miniaturization [1]. The transition towards the $10 \mathrm{~nm}$ technology node [2] poses new challenges to materials scientists and reliability engineers alike. In particular, diffusion barriers that separate the adjacent materials (mostly dielectrics and semiconductors) from the metal interconnect (where $\mathrm{Cu}$ has replaced $\mathrm{Al}$ ) in microelectronics play a critical role and are often decisive for device performance and lifetime [3]. They prohibit the migration of atoms from the $\mathrm{Cu}$ metallization to the surrounding dielectrics or the Si substrate and vice versa, so that no chemical reactions potentially impairing the device functionality can occur [4].

Due to its favorable structural, thermal and electronic properties, TiN is a stateof-the-art diffusion barrier material. However, its performance still has room to be improved to meet the requirements of ultra large scale integration (ULSI) $[5,6]$. According to the 2013 edition of the International Technology Roadmap for Semiconductors, the diffusion barrier thickness must be scaled down below $2 \mathrm{~nm}$ by 2015 and below $1 \mathrm{~nm}$ by 2021. A feasible strategy to achieve this goal might be to tune the microstructure of the established TiN barriers to increase their efficiency [6,7]. Simultaneously, metrology tools must be developed to characterize the atomic structure and composition of the complex materials systems, preferably in 3D. The need to gather local information at nanoscale dimensions on the one hand and to do this over a relatively large area like a $\mathrm{Si}$ wafer on the other hand makes this task even more demanding. A possibility to bridge this gap is to employ numerical modelling and simulation. Still, it remains essential to determine nanoscale material properties as input parameters for metrology models [2].

Transmission electron microscopy (TEM) is a well-established technique that can provide structural, chemical and electronic information with very high spatial resolution. As will be discussed in chapters 2.3 and 4.2, microstructure is a vital factor influencing the performance of diffusion barriers. High-resolution TEM (HRTEM) offers the possibility to acquire lattice resolved images that can be related to the barrier structure. Reciprocal space information can be obtained at the same spatial location and gives further insights into crystallographic orienta- 
tion relationships at interfaces. Moreover, elemental mapping and $Z$ (atomic number)-contrast imaging allow for the visualization of diffusion across the barrier layer [8]. In the past years another technique, atom probe tomography (APT), has gained acclaim as a tool offering true $3 \mathrm{D}$ characterization at the nanoscale. APT is a complementary method to TEM, as it provides 3D compositional imaging and analysis of materials with high sensitivity. Thus, the two techniques enhance each other and together can give information about the morphology, atomic structure and chemical composition of TiN diffusion barrier layers with sub-nanometer resolution.

The aim of the present study is to combine various TEM techniques with APT in order to present a sophisticated and comprehensive analysis approach for the nanoscale investigation of interfaces and interdiffusion processes in model TiN/Cu systems. Paper I focuses on the structural and elemental characterization of the $\mathrm{Cu} / \mathrm{bulk} \mathrm{TiN}$ interface and correlates experimental observations of $\mathrm{Cu}$ diffusion in single-crystal TiN with results of first-principles studies [9]. Paper II compares the diffusion of $\mathrm{Cu}$ in single- and polycrystalline TiN barrier layers. This was taken as a starting point to examine possible atomic $\mathrm{Cu}$ diffusion mechanisms in the single-crystal TiN barrier by density-functional theory (DFT) calculations. The combined approach of state-of-the-art experimental techniques and firstprinciples calculations is in accordance with the metrology needs projected by the International Technology Roadmap for Semiconductors and enables a direct comparison between diffusion of $\mathrm{Cu}$ in TiN films with different microstructures, thus contributing to the understanding of complex diffusion phenomena.

It is my hope that this knowledge can ultimately become the basis for further innovations in ultrathin and highly effective diffusion barrier layers for microelectronics. 


\section{DIFFUSION}

\subsection{The Diffusion Equations}

Diffusion is defined by the Encyclopcedia Britannica as a "process resulting from random motion of molecules by which there is a net flow of matter from a region of high concentration to a region of low concentration" [10]. Although the phenomenon was utilized as early as $500 \mathrm{BC}$, for example in the production of Indian Wootz steel used for the famous Damascus swords [11], it was only beginning to be investigated systematically in the early nineteenth century. The first scientifically relevant treatises on diffusion are commonly attributed to John Dalton [12] and Thomas Graham [13], who both looked into the intermixing behavior of gases [14]. A mathematical basis describing diffusion phenomena was established by Adolf Fick in 1855 [15]. Fick postulated that under the assumption of steady-state conditions, the net material flux $J$ along a direction $x$ is proportional and opposed to the concentration gradient $\partial C / \partial x$ via a proportionality constant called the diffusion coefficient $D$ :

$$
J=-D \frac{\partial C}{\partial x}
$$

This equation is known as Fick's first law [15,16].

Considering that especially solid-state diffusion is a relatively slow process, concentration changes with time $t$ must also be accounted for in the mathematical treatment of diffusion phenomena [17]. Since diffusing particles are neither created nor destroyed in absence of any chemical reactions, a continuity equation can be written as

$$
\frac{\partial C(x, t)}{\partial t}=-\frac{\partial J}{\partial x}
$$

Under the assumption that $D$ is independent of $C$ and $x$, substituting equation (1) into (2) yields Fick's second law [15,16]:

$$
\frac{\partial C(x, t)}{\partial t}=D \frac{\partial^{2} C}{\partial x^{2}}
$$

This equation is often simply referred to as the (linear) diffusion equation [18].

Diffusion is the sole mass transport mechanism in solids [19]. In a crystal, atoms vibrate about their equilibrium positions with a characteristic frequency $v_{0}$ (called the Debye frequency), which is of the order of $10^{13} \mathrm{~s}^{-1}$. This lattice vibration is 
rarely sufficient to cause a direct interchange between two atomic positions; however, if a neighboring lattice site is vacant, a successful jump becomes much more likely. Vacancy formation is governed by the principles of thermodynamics and the fraction $f_{v}$ of vacant sites at a certain temperature $T$ can thus be given as

$$
f_{v}=\exp \left(-\frac{E_{f}}{k T}\right)
$$

with $E_{f}$ as the vacancy formation energy $(\approx 1 \mathrm{eV} /$ atom) and $k$ as the Boltzmann constant. For a jump to occur, the atom also requires energy to overcome the potential barrier between two neighboring lattice sites. The probability $W$ of acquiring the necessary jump or migration energy $E_{m}$ is

$$
W=v_{0} \exp \left(-\frac{E_{m}}{k T}\right)
$$

If two neighboring lattice planes, denoted as 1 and 2, with a lattice constant $a_{0}$, are situated in a region where an atomic concentration gradient exists, so that there are $n_{1}=C a_{0}$ atoms per unit area of plane 1 , and $n_{2}=n_{1}+(d n / d x) a_{0}=$ $\left(C+\left(d C a_{0} / d x\right)\right) a_{0}$ atoms per unit area of plane 2 , the resulting atom fluxes from plane 1 to 2 and vice-versa are given by

$$
\begin{gathered}
J_{1 \rightarrow 2}=\frac{1}{6} v_{0} \exp \left(-\frac{E_{m}}{k T}\right) \exp \left(-\frac{E_{f}}{k T}\right) C a_{0}, \\
J_{2 \rightarrow 1}=\frac{1}{6} v_{0} \exp \left(-\frac{E_{m}}{k T}\right) \exp \left(-\frac{E_{f}}{k T}\right)\left(C+\frac{d C a_{0}}{d x}\right) a_{0} .
\end{gathered}
$$

The factor $1 / 6$ accounts for atomic jumps in positive and negative directions in three dimensions. The net flux can be calculated as the difference $J_{1 \rightarrow 2}-J_{2 \rightarrow 1}$ :

$$
J_{n e t}=-\frac{1}{6} v_{0} a_{0}^{2} \exp \left(-\frac{E_{m}}{k T}\right) \exp \left(-\frac{E_{f}}{k T}\right)\left(\frac{d C}{d x}\right) .
$$

Substituting equation (8) into (1) yields an Arrhenius-type temperature dependence of the diffusion coefficient:

$$
D=\frac{1}{6} v_{0} a_{0}^{2} \exp \left(-\frac{E_{m}}{k T}\right) \exp \left(-\frac{E_{f}}{k T}\right)=D_{0} \exp \left(-\frac{Q}{k T}\right),
$$

with the diffusion activation energy $Q$ as the sum of $E_{m}$ and $E_{f}$. The preexponential or frequency factor $D_{0}$ can be considered a material constant and represents the diffusion coefficient at infinite temperature $[17,18]$. 
This model is not only applicable to lattice diffusion, but can also describe diffusion along grain boundaries or interfaces, if the activation energy is modified accordingly $[16,17]$.

\subsection{Solution of the Diffusion Equations for Thin Films}

The thin film solution of Fick's second law assumes non-steady-state diffusion in one dimension. The diffusing species is deposited in the form of a thin film on a bulk sample surface at $x=0$ and spreads into one half-space for $t>0$. In this case, the initial condition is called instantaneous planar source condition [18] and reads

$$
C(x, t=0)=M \delta(x),
$$

with $M$ as the number of diffusing particles per unit area and $\delta(x)$ as the Dirac delta function [20]. The solution to the diffusion equation is then of a Gaussian form and given by

$$
C(x, t)=\frac{M}{\sqrt{\pi D t}} \exp \left(-\frac{x^{2}}{4 D t}\right)
$$

The diffusion length $\bar{x}$ at which the argument in the exponential function equals -1 is known as characteristic diffusion length and is often encountered in diffusion problems [18]. Since it is closely linked to the random walk during Brownian movement of particles described by Albert Einstein [21], it is sometimes also called Einstein's diffusion path [17]:

$$
\bar{x}=2 \sqrt{D t} .
$$

Thus, most diffusion experiments are designed in such a way that the diffusion time and temperature $T$ are known, and the diffusion length can be measured in order to obtain $D(T)$ from equation (12). This information can then be used to find $D_{0}$ and $Q$ from an Arrhenius plot of equation (9).

\subsection{Diffusion Mechanisms}

\subsubsection{Lattice diffusion}

Atomic movement in solids is restricted by the crystal lattice. Diffusion mechanisms can thus be described in terms of atomic displacements, where the features of the atomic jump process depend on the host crystal structure and the size and 
chemical nature of the diffusing atom. On this basis several lattice diffusion mechanisms have been defined as illustrated in Fig. 1 [18].

- Vacancy mechanism: Atoms generally move through the crystal lattice by a series of exchanges with vacancies. In pure metals and alloys vacancies are present at all temperatures. Near the melting temperature their concentration is about $0.01-0.001 \%$. The jump of an atom into a neighboring vacancy site is the dominant (self-) diffusion mechanism of matrix atoms and substitutional solutes in metals and alloys, and also a number of ceramic materials [18].

- Interstitial mechanism: Small solute atoms like O, N, C, and H occupying octahedral or tetrahedral interstitial positions can diffuse by jumping along neighboring interstitial sites. The jumping process involves reversible lattice straining when the diffusing atom displaces matrix atoms while moving between interstitial sites, but no defect formation is required. Therefore, the diffusion coefficients for the interstitial mechanism are comparably high [18].

- Collective mechanism: In substitutional solid solutions the simultaneous motion of several atoms is required, so that diffusion either occurs via a direct interchange of neighboring atoms or by the rotation of three or more atoms as a group by one atomic distance (ring mechanism). The large lattice distortions associated with these processes make the collective diffusion mechanism energetically unfavorable in most solids with the exception of amorphous systems [18].

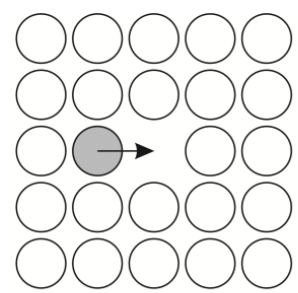

(a) Vacancy mechanism

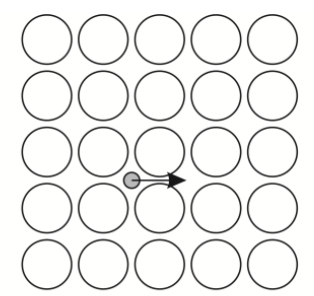

(b) Interstitial mechanism

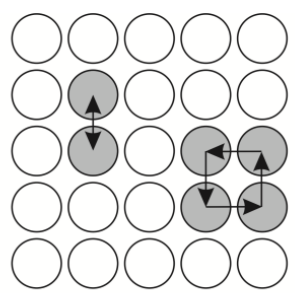

(c) Collective mechanism

Figure 1: Schematic illustration of lattice diffusion via the (a) vacancy, (b) interstitial, and (c) collective mechanism.

\subsubsection{Grain boundary diffusion}

A grain boundary is a transition region between two crystals with different crystallographic orientations. The atomic packing in this transition region is less dense than in the perfect crystal. Therefore, grain boundaries provide preferred pathways for diffusing particles. The internal structures of different grain boundaries can vary significantly, and grain boundary diffusion was found to be anisotropic [22] and dependent on the relative misorientation between adjacent grains $[7,23]$. 
The Arrhenius relation given in equation (9) also holds for the polycrystalline case, if all lattice diffusion terms $\left(D, D_{0}\right.$, and $\left.Q\right)$ are replaced by grain boundary diffusion terms $\left(D_{g b}, D_{0 g b}\right.$, and $Q_{g b}$, respectively). Due to the lower activation energy involved in grain boundary diffusion, $D_{g b}$ is generally several orders of magnitude larger than $D$ [18]. Depending on the interplay between lattice and grain boundary diffusion, three diffusion regimes can be distinguished in polycrystals as illustrated in Fig. 2 [24].

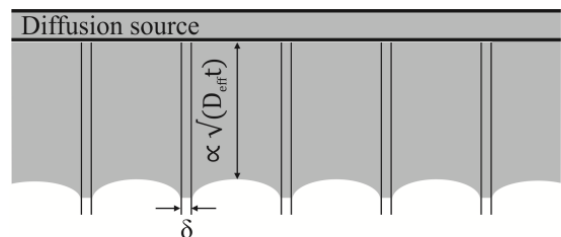

(a) Type A

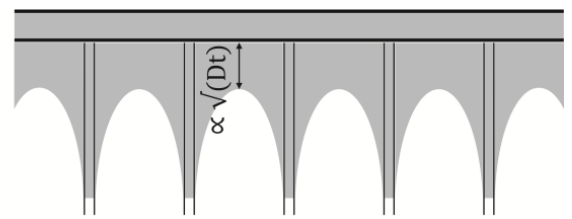

(b) Type B

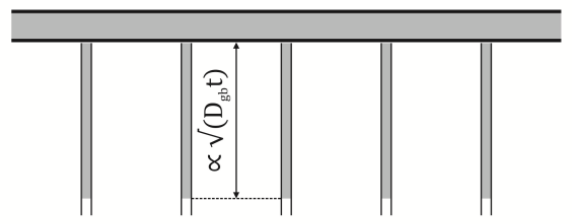

(c) Type C

Figure 2: Diffusion in polycrystals following the (a) type A kinetic regime at high temperatures, (b) type $\mathrm{B}$ regime at intermediate temperatures, and (c) type $\mathrm{C}$ regime at low temperatures.

- Type A diffusion regime: After annealing treatments at high temperatures or for long time spans diffusion in the lattice can mask the penetration along grain boundaries, resulting in an almost planar diffusion front. The effective diffusivity $D_{\text {eff }}$ is a weighted average of the lattice and grain boundary diffusion coefficient. The diffusion length is then proportional to $\sqrt{D_{e f f} t}$.

- Type $B$ diffusion regime: In this intermediate case, diffusion occurs from the grain boundaries into the adjacent grains, leading to the development of distinct lattice diffusion fringes. While the lattice diffusivity $D$ can be estimated from equation (12), mathematical analyses by Whipple [25] and Suzuoka [26] have shown that the grain boundary diffusion coefficient $D_{g b}$ cannot be calculated independently of the grain boundary width $\delta$ by solving the diffusion equations within the grain boundaries. Only the product $\delta D_{g b}$ is accessible. A grain boundary width $\delta=0.5 \mathrm{~nm}$ is therefore often assumed to obtain $D_{g b}$, which has since been proven to be a good estimate $[27,28]$.

- Type $C$ diffusion regime: No significant lattice diffusion occurs at annealing treatments at low temperatures or for very short periods due to insufficient activation energies. Diffusion is limited to the grain boundaries, where the diffusion length is proportional to $\sqrt{D_{g b} t}$. 



\section{FILM SYNTHESIS}

\subsection{Magnetron Sputter Deposition}

Magnetron sputtering belongs to the class of physical vapor deposition (PVD) techniques. As all deposition processes it can be divided into three steps: (i) the transition of the material to be deposited from the condensed phase to the vapor phase, (ii) the transport of the vapor from the source to the substrate, and (iii) condensation of the vapor at the substrate, followed by film nucleation and growth [29]. In contrast to the thermal evaporation of a material utilized in other PVD processes, sputtering involves the physical evaporation of atoms by momentum transfer from impinging energetic particles [30].

A schematic of the sputtering system used to deposit the films studied within the present thesis is shown in Fig. 3. It consists of a vacuum chamber, equipped with three circular planar cathodes (often referred to as targets). Each target is connected to a separate power supply. Opposite the targets the substrates are mounted on a rotating holder, which can be heated up to $700{ }^{\circ} \mathrm{C}$. Additionally, a DC or pulsed bias potential can be applied to the substrate holder. The deposition chamber further provides several feed-throughs, which are used as gas inlets and ducts to the vacuum pump (not shown).

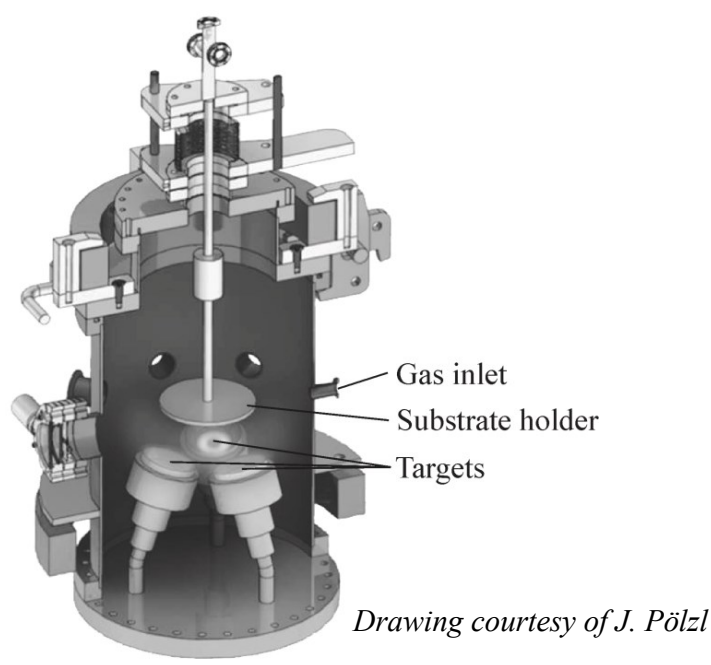

Figure 3: Section through a laboratory-scale magnetron sputter deposition chamber. 
A typical sputter deposition process proceeds as follows: The sputtering system is evacuated to a base pressure in the high or ultra-high vacuum range. Subsequently, the working gas - usually an inert gas like $\mathrm{Ar}$ - is introduced into the chamber. A negative voltage is applied to the targets which serve as cathodes, while the grounded or biased substrate holder becomes the anode. Stray electrons present in the working gas are accelerated towards the anode and collide with neutral gas atoms, thereby transforming them into positively charged ions. Due to charge conservation this impact ionization process in turn releases two electrons, as shown using the example of $\operatorname{Ar}[17,30,31]$ :

$$
\mathrm{Ar}+\mathrm{e}^{-} \rightarrow \mathrm{Ar}^{+}+2 \mathrm{e}^{-}
$$

On the other hand, ions are accelerated towards the cathode, where they impinge on the target and eject secondary electrons. These electrons are now available for impact ionization processes as well, resulting in a snowball effect, and a selfsustaining gas discharge is initiated. This partially ionized, quasi-neutral gas is known as plasma [17].

Once the glow discharge is established, material transfer from the target to the substrate is observable. Energetic particles striking a surface can cause a number of different effects, amongst them the aforementioned emission of secondary electrons. Most importantly for the sputtering process, bombardment with energetic particles leads to the ejection of target atoms, under the condition that the energy transferred in the collision is sufficient to overcome the atom's surface binding energy. The atom can be sputtered due to a single knock-on event, or as a result of a collision cascade [17,31]. In addition to the energy, the sputter yield (the ratio of incident ions to sputtered atoms) also depends on the mass of the bombarding ion and the target material. Most practical sputtering processes achieve a sputter yield of 0.1-10 [17].

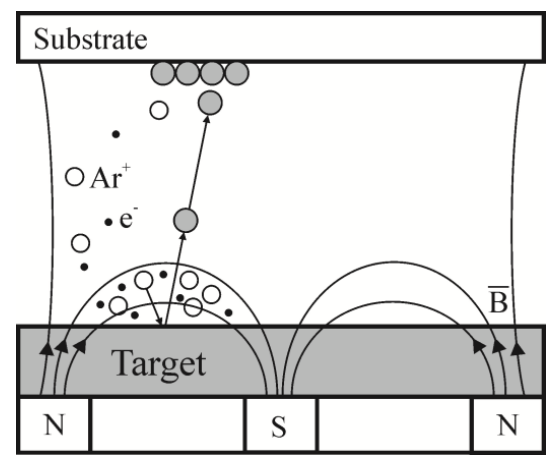

Figure 4: Illustration of the sputtering process occurring in an unbalanced magnetron configuration. 
The deposition rate can be increased if secondary electrons are trapped near the target by a magnetic field. A suitable arrangement of magnets for a planar magnetron is shown in Fig. 4. Electrons are confined to a closed path above the target, creating a high-density plasma. A visual manifestation of the locally enhanced ionization of the working gas is a pronounced erosion zone ("racetrack") evident on the used target. However, the complete confinement of the plasma to the target surface is not ideal, since ions are often also utilized to modify film growth at the substrate. Therefore, in most technical applications a so-called unbalanced magnetron configuration is used, where the magnetic field is weakened selectively to allow some secondary electrons to escape from the target region. Magnetic field lines appropriate to this set-up are also plotted in Fig. 4 $[17,32,33]$.

Sputter deposition of compounds such as TiN can either be achieved by sputtering from compound targets or sputtering in the presence of a reactive gas [34]. The TiN films discussed in the present thesis were deposited in a mixed $\mathrm{Ar} / \mathrm{N}_{2}$ discharge by sputtering pure Ti targets. The possibility of reactive sputtering makes the process more versatile but also more complex, since additional effects such as compound formation on the target surface ("poisoning") have to be taken into account [35].

\subsection{Film Nucleation and Growth}

\subsubsection{Nucleation and early stages of growth}

Once atoms have been sputtered from the target they travel through the plasma, where they may interact with the present plasma species, and eventually reach the substrate [29]. Sputtered atoms typically have a kinetic energy in the range of $5-10 \mathrm{eV}$, which is comparable to the bond energy in solids. Even with this relatively high energy, it only takes a few vibrational periods for the incident atoms to become thermally accommodated at the substrate. Adsorbed atoms (adatoms) then diffuse along the substrate surface until they reach an energetically favorable position or are desorbed again. The surface diffusion is governed by the substrate temperature and kinetic energy of the adatoms as well as by the involved materials. Preferred nucleation sites on the substrate include lattice defects and atomic steps. Fig. 5(a) depicts the processes occurring in the early stages of nucleation. Once a nucleus exceeds a critical size determined by the trade-off between volume free energy and surface energy, it is said to be stable. Stable nuclei continue to grow by the incorporation of further adatoms and subcritical clusters, forming distinct islands [17,36]. Eventually, these islands coalesce with their neighbors, thus gradually decreasing the overall island density. Channels and voids in the coalesced network are filled by continued atom influx, finally resulting in a continuous film. These processes account for the first few hundred Ångströms of film thickness [17]. 


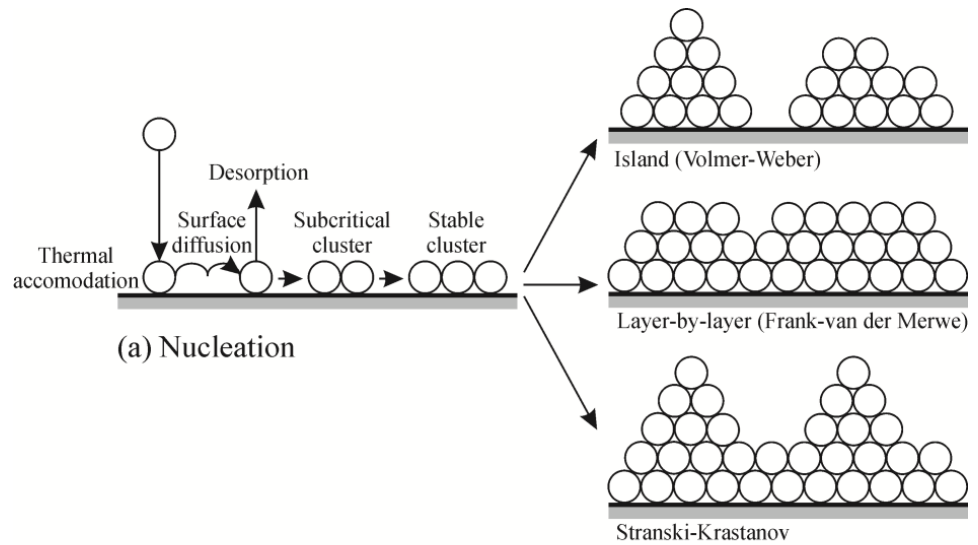

(b) Growth

Figure 5: Schematic representation of (a) the steps leading to nucleation and (b) the three film growth modes.

Subsequent film growth can be classified according to three different growth modes, schematically illustrated in Fig. 5(b).

- Island (Volmer-Weber) growth is a three-dimensional growth mode. It occurs when the net surface free energy associated with cluster formation is positive, indicating that deposited atoms are more strongly bound to each other than to the substrate $[17,36]$. Island growth is often observed when depositing metals and semiconductors on gas-metal-compounds such as $\mathrm{SiO}_{2}, \mathrm{NaCl}$, or $\mathrm{TiO}_{2}$ [37-39].

- Layer-by-layer (Frank-van der Merwe) growth is a two-dimensional growth mode. If atoms in the deposit are more strongly bound to the substrate than to each other, a stable nucleus will grow in a planar fashion. The layer growth mode is sustained provided there is a continuous decrease in bonding energy between the layers from the first monolayer to the bulk-crystal value $[17,36]$. A classic example of layer growth is contamination-free homoepitaxial growth of semiconductor films [40], but it also occurs when depositing a low-meltingpoint metal on a high-melting-point metal [41].

- Stranski-Krastanov growth develops when layer growth becomes unfavorable after the formation of one or more monolayers and island growth proceeds. This is a fairly common growth mode and often occurs as a strain relaxation mechanism (strain-induced roughening) [17,36]. Stranski-Krastanov growth has been observed on metal-metal, metal-semiconductor, and semiconductorsemiconductor systems [42-44].

Examples for all of these growth modes can be pointed out within the present study. Single-crystal growth of $\mathrm{TiN}$ on $\mathrm{MgO}$ has been found to occur in a layerby-layer mode [45], which is reasonable also given the minute lattice mismatch 
between the two materials (see chapter 3.2.2). While no in-situ measurements have been carried out in the study at hand to directly observe the growth mode of $\mathrm{TiN}$ on $\mathrm{MgO}$, the $\mathrm{X}$-ray reflectivity investigations reporting a TiN surface roughness in the range of the $\mathrm{MgO}$ substrate roughness in Paper I are a good argument in support of layer-by-layer growth. Literature commenting on the growth mode of $\mathrm{Cu}$ on single-crystal TiN is scarce, but HRTEM examinations (again see chapter 3.2.2 and Fig. 6) point towards a form of Stranski-Krastanov growth also as a possible strain relaxation mechanism. An example for the three dimensional island growth mode is polycrystalline $\mathrm{TiN}$ on $\mathrm{Si}$ [46].

Aside from these basic growth modes determined by the interaction between deposit and substrate, there is also a significant influence of the substrate temperature and deposition rate on nucleation and early growth processes. In general, higher substrate temperatures increase the size of the critical nucleus, while the number of stable nuclei is reduced. On the other hand, a higher deposition rate leads to smaller islands, since adatoms and clusters do not have sufficient time to diffuse before they are buried by subsequent incident atoms. The nucleation rate in turn is increased, so that a continuous film forms at lower film thickness. Thus, deposition at high substrate temperatures and low deposition rates promotes the growth of large grains or even single-crystals, while low substrate temperatures and high deposition rates favor the formation of polycrystalline films [17]. Film microstructure will be discussed in more depth in the following sections.

\subsubsection{Epitaxial growth of single-crystalline films}

The word epitaxy has its roots in ancient Greek and can be roughly translated as "arranged upon". It generally refers to a film/substrate system with a defined crystallographic orientation relationship but is often used synonymously with single-crystal film growth on a crystalline substrate. The most important parameter characterizing epitaxy is the lattice mismatch $\bar{f}$ between the film and substrate material $[17,47]$.

$$
\bar{f}=\frac{a_{0}(s)-a_{0}(f)}{a_{0}(f)} \times 100 \%,
$$

where $a_{0}(s)$ and $a_{0}(f)$ are the unstrained lattice parameters of the substrate and film, respectively. As a rule of thumb, epitaxial growth requires a lattice mismatch of less than $15 \%$ [47]. If $\bar{f}$ equals zero, the substrate parameters are perfectly matched. This occurs in the growth of homoepitaxial layers, i.e. layers that are of the same material as the substrate. A popular example is the growth of high-purity Si films on Si wafers [48]. If the film and substrate are composed of different materials, this is referred to as heteroepitaxy. In this case the epilayers are either strained or compressed to accommodate the lattice misfit, or relaxed through the formation of misfit dislocations at a critical film thickness [47]. 
Single-crystalline $\mathrm{TiN} / \mathrm{Cu}$ layers investigated within the present thesis have been grown epitaxially on (001)-oriented $\mathrm{MgO}$ substrates. The growth of epitaxial TiN on $\mathrm{MgO}$ by magnetron sputtering is widely reported in literature [49-55]. Both materials crystallize in the $\mathrm{B} 1$ face centered cubic (fcc) $\mathrm{NaCl}$ structure and have a minimal lattice mismatch of $-0.7 \%[56,57]$. In comparison, the lattice mismatch between $\mathrm{TiN}$ and $\mathrm{fcc} \mathrm{Cu}$ amounts to $-17 \%[57,58]$, thus slightly exceeding the $15 \%$ rule. Still, epitaxial growth of $\mathrm{Cu}$ on $\mathrm{TiN}$ is not an unreasonable assumption, as it has been reported previously for $\mathrm{Cu}$ films deposited on $\mathrm{MgO}$, where the lattice mismatch is comparable [59,60]. Pole figure measurements and TEM investigations discussed in the appended Paper I confirm that the TiN/Cu bilayers exhibit a cube-on-cube epitaxial relationship with the substrate, i.e. $\{001\}<010>\mathrm{TiN} / \mathrm{Cu} \|\{001\}<010>\mathrm{MgO}$ [9].

Fig. 6(a) shows a high-resolution TEM image of the pristine TiN/Cu interface. The measured lattice spacing of the $\mathrm{Cu}(020)$ planes is $0.182 \mathrm{~nm}$. Compared to the unstrained value of $0.181 \mathrm{~nm}$ [58], the $\mathrm{Cu}$ layer appears only minimally strained along [010]. This indicates that most of the interface strain associated with the lattice mismatch is relieved through the incorporation of dislocations in the near interface region, as evident in Figs. $6(\mathrm{~b}, \mathrm{c})$. The measured $(020)$ lattice spacing in TiN is $0.202 \mathrm{~nm}$ (unstrained spacing $0.212 \mathrm{~nm}$ [57]). As expected from the lattice mismatch to $\mathrm{MgO}$, TiN is compressed along the [010] direction. It has to be noted that neither the influence of the TiN stoichiometry nor of differential thermal expansion during the deposition process has been taken into account in the above discussion. Especially the latter may contribute to a more

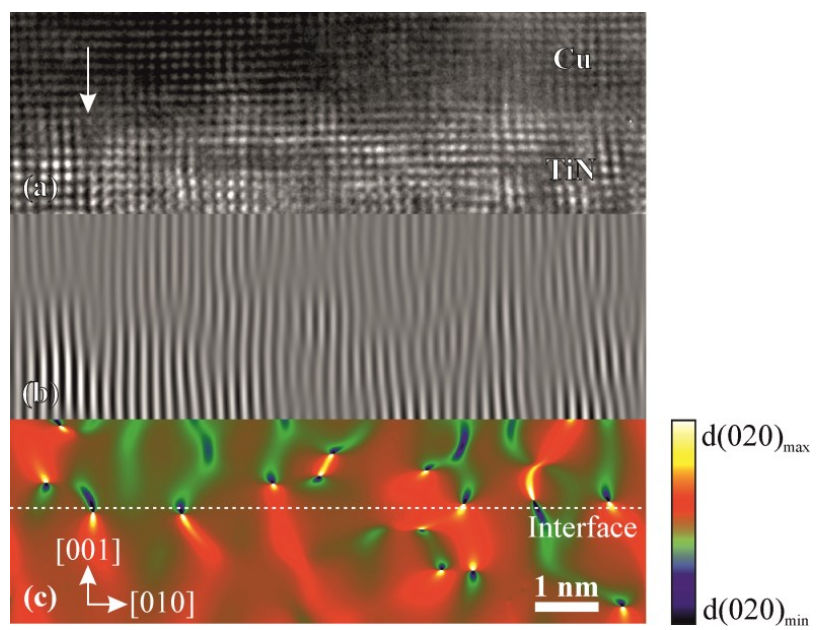

Figure 6: (a) Cross-sectional high-resolution TEM image of the $\mathrm{TiN} / \mathrm{Cu}$ interface recorded along the [100] zone axis with an exemplary dislocation marked by the arrow, (b) corresponding Bragg image showing the (020) planes and (c) corresponding qualitative map of the distribution of the interplanar spacing $\mathrm{d}(020)$. 
complex stress/strain state in the bilayers, as can be understood when considering the following example. The investigated TiN barrier layers are deposited on $\mathrm{MgO}(001)$ at a substrate temperature of $700{ }^{\circ} \mathrm{C}$. The $\mathrm{TiN} / \mathrm{MgO}(001)$ system is then cooled down to $50^{\circ} \mathrm{C}$ for the $\mathrm{Cu}$ deposition. In this case, differential contraction during cooling will introduce compressive stresses of up to $2.22 \mathrm{GPa}$ in the (001)-oriented TiN epilayers in addition to the compression due to the lattice mismatch to $\mathrm{MgO}$ [61]. Therefore, when depositing epitaxial films at high substrate temperatures, a close match not only between the lattice parameters but also between the thermal expansion coefficients of the materials is desirable [17].

\subsubsection{Polycrystalline films}

Polycrystalline thin films exhibit diverse microstructures, which can be characterized in terms of grain size, crystallographic orientation, defects, composition and morphology [36]. As PVD material synthesis frequently proceeds far from thermodynamic equilibrium, kinetic limitations control film growth. Surface and bulk diffusion are the main atomic processes determining grain shape and orientation, which often develop in a competitive fashion. Tuning deposition parameters such as substrate temperature, additional ion irradiation, or partial pressures of reactive gases thus allows for the adjustment of microstructural features in engineering thin films [36,62]. A guideline for microstructural design of polycrystalline PVD coatings is provided by structure zone models (SZMs), which systematically categorize the structural evolution as a function of deposition parameters.

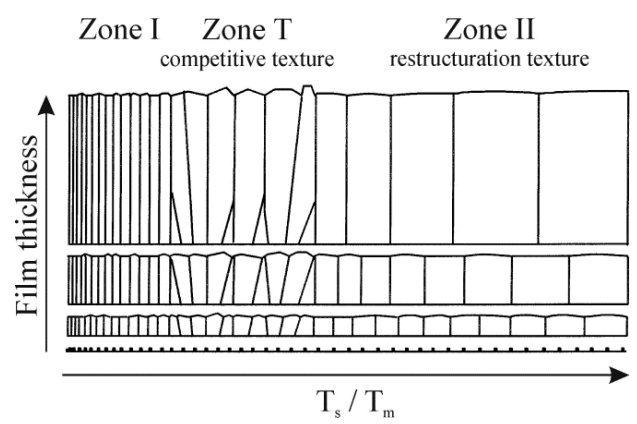

Figure 7: Basic structure zone model for elemental films adapted from Barna and Adamik [62].

The basic structure zone model for elemental films as depicted in Fig. 7 illustrates film growth regimes with increasing temperature and film thickness disregarding any effects of impurities. The parameter plotted on the x-axis is the homologous temperature, the ratio of the substrate temperature $T_{s}$ to the melting temperature of the film $T_{m}$ (both in Kelvin). Depending on the homologous temperature, the SZM is comprised of three regions [17,36,62]: 
- Zone I $\left(0<T_{s} / T_{m}<0.2\right)$ : In this low temperature growth regime adatom diffusion is negligible. The resulting fiber structure is underdense featuring extensive inter- and intracolumnar porosity. The nucleation density determines the lateral fiber dimensions, and the random orientation of the nuclei translates into the orientation of the fibers.

- Zone $T\left(0.2<T_{s} / T_{m}<0.4\right)$ : Surface diffusion of adatoms is significant in the transition zone growth regime. The film structure develops through competitive growth of adjacent crystals with different orientations, resulting in typical $\mathrm{V}$-shaped grains close to the nucleation layer and culminating in a columnar morphology at higher film thicknesses. With increasing temperature also grain boundaries become more mobile and the evolution of a preferred orientation can be observed in the higher temperature part of the transition zone. Facetted column tops give rise to a significant surface roughness. The resulting shadowing effects often lead to the formation of underdense column boundaries.

- Zone II $\left(0.4<T_{s} / T_{m}\right)$ : In this growth regime the contributions of bulk diffusion and grain boundary migration become significant. The driving force for orientation selection during grain coalescence and coarsening is the minimization of interface and surface energy. If grains are strongly textured, abnormal grain growth may occur, resulting in large columnar grains with a monomodal grain size distribution [63]. The lateral dimensions of the columns increase with increasing homologous temperature.

This SZM by Barna and Adamik is an example of the multiple models proposed in literature and has been presented here in more detail due to its wide applicability. Barna and Adamik expanded their model in the same paper to account for the influence of impurity species, which they argued are unavoidable in thin film synthesis. Impurities typically segregate at grain boundaries, where they inhibit grain growth, leading to the formation of less textured films with smaller column dimensions, or - in extreme cases - to films composed of randomly oriented globular grains [62]. Thornton developed a SZM with an additional axis taking the sputtering gas into consideration, but found little influence of the working gas pressure especially at higher temperatures [64]. Similarly, Anders incorporated the impact of plasma and ion irradiation on film growth in a SZM, describing the displacement and heating effects caused by the kinetic energy of bombarding particles [65].

\subsubsection{Effects of ion irradiation}

Providing energy in the form of moderate ion bombardment to the film forming species promotes the adatom surface diffusion and the chemical reactivity in case of reactive deposition. Beneficial effects include increased film density, modified grain morphology (e.g. a transition from columnar to equiaxed growth), enhanced surface smoothness and the possibility to manipulate film texture and tune stress levels $[17,36,66]$. Ideally, the ion flux reaching the substrate should be 
high, while the ion energy should be low $(<10-20 \mathrm{eV})$ to avoid damaging the film [36]. A simple and frequently used approach is to apply a negative bias voltage to the substrate to vary the ratio of incident ions to neutrals at the growing film. In this study, TiN barrier layers are deposited at an asymmetrically pulsed DC substrate bias of $-100 \mathrm{~V}$. Bipolar pulsing of the bias voltage is necessary to accommodate the electrically non-conductive $\mathrm{MgO}$ substrates. The $\mathrm{DC}$ voltage is pulsed at a frequency of $250 \mathrm{kHz}$ with a positive pulse duration of $496 \mathrm{~ns}$ at a preset amplitude of $+37 \mathrm{~V}[67]$.

Ion irradiation has been shown to increase the N-content in TiN films up to the stoichiometric composition [68]. Moderate substrate biasing also leads to a densification of grain boundaries through collision cascade effects and a reduction in grain size due to continued renucleation at ion induced point defects [69]. The promotion of a (002) preferred growth orientation in polycrystalline TiN has been attributed to ion irradiation as well. Together with the higher density this significantly enhances the mechanical performance of TiN coatings [70]. However, if the bias voltage is chosen too high, detrimental effects such as increased intragranular defect densities and entrapment of noble gas ions become crucial $[50,69]$.

Therefore, a precise adjustment between substrate temperature and applied bias voltage is necessary to ensure optimum growth conditions depending on the eventual application of the TiN layer. In the present study this was realized by performing a preliminary deposition series of $\mathrm{TiN}$ on $\mathrm{MgO}(001)$ and $\mathrm{Si}(001)$ at a substrate temperature of $700{ }^{\circ} \mathrm{C}$ with a bias voltage variation from 0 to $-200 \mathrm{~V}$ in $50 \mathrm{~V}$ steps. The resulting films were assessed by X-ray diffraction and scanning electron microscopy (SEM). While no orientation changes were observable in the diffractograms of TiN grown on either of the two substrate materials, a decrease in deposition rate was evident at bias voltages of -150 and $-200 \mathrm{~V}$. This indicates that significant resputtering was taking place during film growth. The TiN films grown on $\mathrm{Si}$ at 0 and $-200 \mathrm{~V}$ also exhibited voids in cross-sectional SEM images. Therefore, a bias voltage of $-100 \mathrm{~V}$ was chosen as the optimum setting at $T_{S}=700{ }^{\circ} \mathrm{C}$. This is in good agreement with previous experiments carried out in the same deposition system, which found identical deposition parameters to result in the growth of low-defect TiN films [71]. 



\section{FUNCTIONAL TITANIUM NITRIDE FILMS}

\subsection{Structure and Properties of TiN}

\subsubsection{Composition, structure and mechanical properties}

TiN belongs to the group of refractory interstitial transition metal nitrides. The melting temperature of the stoichiometric compound is $2949^{\circ} \mathrm{C}$ and it crystallizes in the fcc B1 structure [72]. The binary TiN phase is stable over a wide composition range [73]. Therefore, the intrinsic defect density in TiN can become very high, with vacancies and interstitials both on the Ti- and the $\mathrm{N}$ sublattices. The properties of TiN depend strongly on its actual composition. This effect is even more pronounced in thin films, since they are normally deposited under non-equilibrium conditions [74].

One of the earliest comprehensive investigations of sputtered TiN coatings was carried out by Sundgren et al. $[68,74-76]$. They determined that the ratio of reactive to sputtering gas as well as the ion current density at the substrate and the voltage applied to the target are the most influential process parameters dictating the TiN film composition [68]. Cross-sectional electron micrographs reveal that sputtered stoichiometric polycrystalline TiN grows with a dense columnar structure classified as zone $\mathrm{T}$ in the SZM. At a Ti/N ratio of one, TiN films have the full bulk density of $5.39 \mathrm{~g} \mathrm{~cm}^{-1}$ and exhibit a minimum electrical resistivity of $25 \mu \Omega \mathrm{cm}$ [75]. Similarly, the lattice parameter reaches its maximum value of $4.242 \AA$ at the stoichiometric composition and decreases for both over- and understoichiometric films $[74,75]$. As discussed in chapter 3, the microstructure of sputtered TiN can also be controlled by using different substrate materials. Epitaxial TiN of high single-crystalline quality can be grown readily on $\mathrm{MgO}$ substrates as shown in Fig. 8(a), since the lattice parameters of fcc TiN and fcc $\mathrm{MgO}$ are almost identical. In contrast, growth on Si substrates or the native oxide typically leads to a fine grained nucleation layer close to the interface, morphing into the characteristic polycrystalline transition zone microstructure with a more or less dense array of cone-shaped columnar grains as can be seen in Fig. 8(b). There are also studies available reporting the pulsed laser deposition of nanocrystalline TiN (at low growth temperatures) and single-crystalline TiN on Si by domain matching epitaxy [6,77-79].

The microstructure has a strong influence on the mechanical performance of TiN films. Patsalas et al. [80] compared TiN films grown at different bias potentials and temperatures on Si. From nanoindentation measurements they found that 


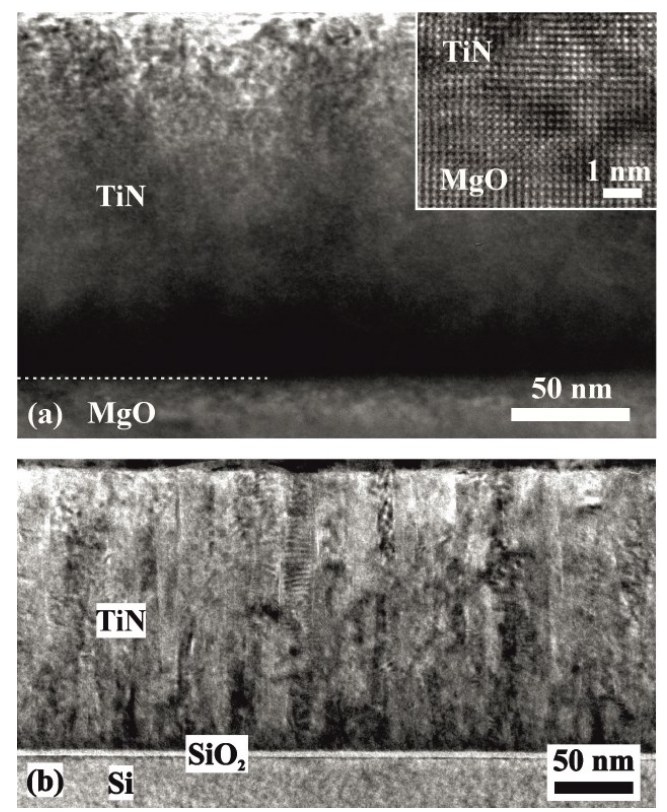

Figure 8: Cross-sectional TEM images of sputter deposited (a) featureless single-crystal TiN grown on $\mathrm{MgO}$ and (b) polycrystalline TiN grown on thermally oxidized $\mathrm{Si}$. The inset in (a) shows a lattice resolved micrograph recorded directly at the TiN/MgO interface along the [100] zone axis, though the exact interfacial planes cannot be determined due to the close lattice match. The polycrystalline TiN depicted in (b) exhibits a typical columnar zone T microstructure.

over a bias potential range from -20 to $-100 \mathrm{~V}$ the film hardness and elastic modulus increase from 17 to $24 \mathrm{GPa}$ and 210 to $320 \mathrm{GPa}$, respectively. The same trends were observed by Shojaei et al. [81]. This behavior can be related to the increased film density and reduction of voids in the films grown under ion irradiation as discussed in chapter 3.2.4. In case of single-crystal TiN, hardness and elastic modulus are dependent on the film orientation. In general, (111)-oriented coatings perform better than (001)-oriented ones, and $\operatorname{TiN}(011)$ performs worst. Ljungcrantz et al. found a hardness of $21 \mathrm{GPa}$ and Young's modulus of $450 \mathrm{GPa}$ for the best $\mathrm{TiN}(111)$ coating and also recognized the possibility of tuning mechanical properties via point defects in single-crystals [51].

\subsubsection{Electronic and optical properties}

TiN has nine valence electrons per atom pair. Eight of those fill bonding states while the additional electron occupies a non-bonding state. This does not modify the bonding properties significantly, but it does make the compound metallic [82]. The electrical resistivity increases with increase of temperature, showing a typical metallic behavior. In single-crystalline $\mathrm{TiN}$ the electrical resistivity at room temperature is $18 \mu \Omega \mathrm{cm}$ [55]. Resistivities of polycrystalline films are typ- 
ically in the range of $25-1000 \mu \Omega \mathrm{cm}[75,83-85]$. Such an increase in resistivity from a single-crystal to a polycrystalline film is due to scattering from the grain boundaries, vacancies and possibly also oxynitrides associated with the polycrystalline TiN $[83,86]$. Moreover, there is also an influence of the layer thickness and grain size on the resistivity. If the films are thinner or the grains are smaller, then scattering of conduction electrons at surfaces or interfaces will add to the overall resistivity [83].

The optical properties of TiN are closely related to the electronic properties. Typical TiN layers with resistivities below $100 \mu \Omega \mathrm{cm}$ have a golden yellow color, while films with increasing resistivities appear bronze to brown. A reflection edge in the visible region with a characteristic reflectivity minimum at about $450 \mathrm{~nm}$ is responsible for the golden yellow color of the pure, stoichiometric TiN, making it a popular choice for decorative coatings [85,87]. The location of the reflection edge can be affected by changes in the carrier concentration. This is caused for example by variations in composition, since TiN can exist with the fcc structure over a wide stoichiometry range. TiN containing excess nitrogen appears bronze to brown in color while substoichiometric TiN is reported to be bright yellow. Other reasons for a shift of the reflection edge include lattice defects, and oxygen and carbon impurities $[85,88]$. However, the color and spectral reflectivity of TiN films can also depend on surface roughness and near-surface defects, arising from the columnar growth in polycrystalline TiN. Comparisons with the electrical properties point toward an inverse correlation of reflectivity and resistivity, with the highest reflectivity and lowest resistivity observed for higher deposition temperatures and moderate ion energies [85].

\subsection{TiN Films as Diffusion Barriers}

\subsubsection{Barrier requirements}

Thin film barrier layers are employed to separate materials and prevent a reaction between them [17]. In microelectronic devices such as ICs, a broad variety of materials, ranging from metals and semiconductors to insulators, are in contact with each other. Intermixing and interdiffusion of these materials and the resulting loss of functionality of the device are a constant concern for manufacturers and often determine device lifetime [89]. Barrier layers must fulfil certain requirements and must be compatible with the surrounding materials. A catalogue of desired characteristics has been compiled by Nicolet [90,91]:

- The material transport rate across the barrier should be negligible.

- The barrier should be thermodynamically stable with respect to the surrounding materials.

- The barrier should adhere strongly to the surrounding materials.

- Contact resistance between the layers should be small. 
- The barrier layer should be uniform in structure and thickness and resistant to mechanical and thermal stress.

- The barrier material should be a good electrical and thermal conductor.

A milestone in IC design was the replacement of the conventional Al (metallization) $/ \mathrm{SiO}_{2}$ (dielectric) technology with $\mathrm{Cu}$ metallizations in combination with low- $k$ (where $k$ stands for the dielectric constant) materials [4,92,93]. By reducing both the resistivity of and capacitance between the interconnects, signal delay times are lowered significantly [3,92]. Especially $\mathrm{Cu}$ and $\mathrm{Si}$ are very reactive even at temperatures as low as $200{ }^{\circ} \mathrm{C}$, and the insertion of a barrier layer between the metallization and the surrounding materials as illustrated in Fig. 9 is paramount to ensure functionality of the device [4]. Thus, with the advent of ULSI, IC dimensions on the nm-scale and the replacement of Al metallization by $\mathrm{Cu}$, additional requirements placed upon diffusion barriers have emerged $[4,89]$ :

- The barrier should be less than $5 \mathrm{~nm}$ thick (for high-resistivity barriers), or should have an electrical conductivity similar to $\mathrm{Cu}$.

- The material must meet advanced processing demands (including uniform deposition of trench walls and bottoms and anisotropic dry-etching).

Naturally, also the cost-benefit ratio plays an important role when developing diffusion barrier materials and the feasibility of reliable and reproducible fabrication without disproportionate expenses must always be considered as a factor [89].

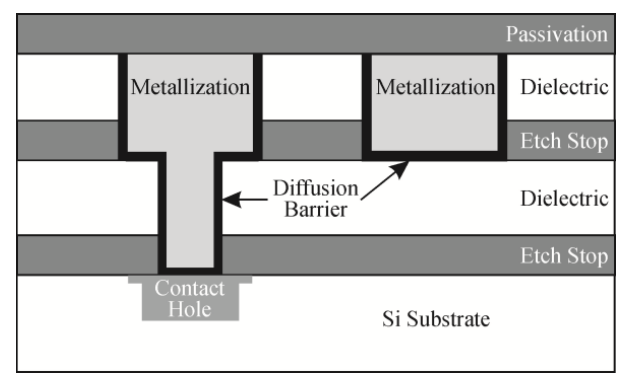

Figure 9: Schematic IC cross-section illustrating the application of diffusion barriers between the metallization and surrounding materials in microelectronic devices.

\subsubsection{Developments in TiN diffusion barrier design}

Sputtered TiN films are well suited as diffusion barrier materials due to their high thermal and structural stability combined with a low electrical resistivity. The latter point is especially important to fully exploit the conductivity advantage of $\mathrm{Cu}$ over $\mathrm{Al}$ interconnects. While many transition metal nitrides fulfil the structural and thermal requirements for effective diffusion barriers, TiN is the material with by far the lowest electrical resistivity. The reported value for the pure polycrystalline film is $20-25 \mu \Omega \mathrm{cm}$, in comparison to up to $200 \mu \Omega \mathrm{cm}$ for TaN and more than $1000 \mu \Omega \mathrm{cm}$ for $\mathrm{WN}$, its strongest contenders [3,75,89]. 
Microstructure plays a critical role in diffusion barrier design. Single-crystalline TiN barriers would provide the perfect solution to the continued search for more efficient and reliable barrier materials due to the lack of fast diffusion paths in the form of grain boundaries. However, constraints in fabrication and processing, such as the lattice mismatch to the substrate and thermal budget limitations, have prohibited the successful industrial implementation of such barrier layers as yet [3]. Paper I emphasizes the excellent performance of single-crystalline TiN barriers, which are demonstrated to withstand annealing temperatures of $900{ }^{\circ} \mathrm{C}$ and show only limited $\mathrm{Cu}$ diffusion after annealing at $1000^{\circ} \mathrm{C}[9]$.

On the other hand, polycrystalline TiN diffusion barrier layers are frequently applied in industry and widely studied in literature. Already in the early 1980s, Chamberlain showed that interdiffusion between r.f. sputtered polycrystalline $\mathrm{Cu}$ and TiN layers at temperatures up to $600-700{ }^{\circ} \mathrm{C}$ is very limited and presumably occurring via grain boundary or dislocation mechanisms [94]. The reason for failure is not barrier dissociation by a chemical reaction, since Ti is bonded with $\mathrm{N}$ and therefore not freely available to react with $\mathrm{Cu}$, but the formation of an intermetallic compound of $\mathrm{Cu}$ and $\mathrm{Si}$ after the diffusion of $\mathrm{Cu}$ along grain boundaries or weak spots in TiN [89]. As a consequence, a promising approach to improve the TiN barrier performance is densification of grain boundaries. This can be achieved by so-called grain boundary stuffing by facilitating the formation of oxides [95-98]. However, this effect is limited to Al metallization, since the formation of $\mathrm{Cu}$-oxides at the TiN grain boundaries is thermodynamically not favorable $[98,99]$. In addition, grain boundary stuffing leads to a resistivity increase in the TiN layer. It has been identified as the primary cause of the high spread of resistivity values for polycrystalline TiN reported in literature [89].

Another strategy is to tune the microstructure of the TiN barrier layer by varying deposition parameters such as substrate temperature and bias potential [6]. However, it has to be considered that this will also influence the overall film density, as discussed in chapter 3.2. The film density is the decisive factor determining the failure temperature of the barrier, if stoichiometry and microstructure of the TiN films is comparable [100]. Therefore, Paper II presents a comparison of diffusion of $\mathrm{Cu}$ in single- and polycrystalline TiN barrier layers deposited at the same substrate temperature of $700{ }^{\circ} \mathrm{C}$ under a bias voltage of $-100 \mathrm{~V}$, sufficient to obtain dense films. For the first time, this allows for a direct comparison of diffusion phenomena and mechanisms in two TiN coatings, which differ from each other only in the presence of grain boundaries. 



\section{TRANSMISSION ELECTRON MICROSCOPY}

\subsection{Interactions of Electrons with Matter}

TEM encompasses a diversity of different techniques that provide information on the structure, topology, morphology, elemental composition, and chemical state of materials [101]. The basis for measurements is the interaction of a high-energy electron beam with a sufficiently thin specimen. Compared to neutrons and photons, electrons interact more strongly with matter, and give rise to a multitude of signals to be interpreted as summarized in Fig. 10 [8].

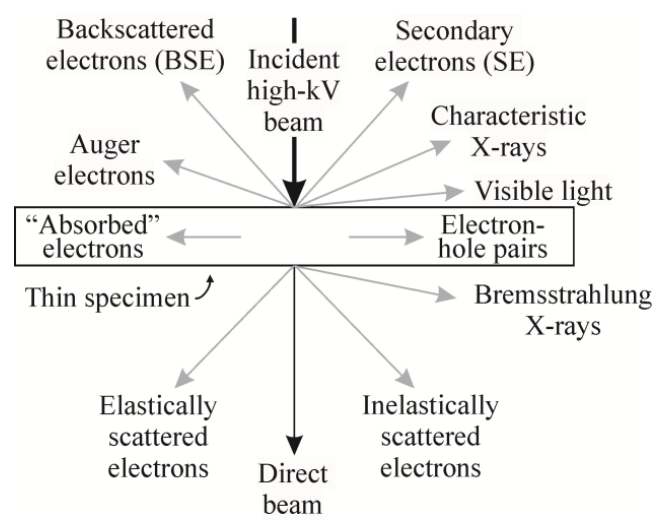

Figure 10: Signals arising from the interaction of a high-energy electron beam with a thin specimen.

The incident electron beam can be considered coherent, meaning that the electron waves are in phase and of a fixed wavelength $\lambda$, which is determined by the acceleration voltage $V$ (relativistic effects must be taken into account) [102]:

$$
\lambda=\frac{h}{\sqrt{2 m_{0} e V\left(1+\frac{e V}{2 m_{0} c^{2}}\right)}} .
$$

Here, $h$ is Planck's constant, $m_{0}$ the electron rest mass, $e$ the elementary charge, and $c$ the velocity of light.

The direct beam contains electrons that pass the sample without any interaction. On the other hand, electrons are scattered elastically due to Coulombic interaction with the electron cloud or nucleus of a sample atom. In this case, no energy 
is transferred from the electron to the sample. Under the condition that the specimen is thin and crystalline, elastic scattering is usually coherent and occurs at low angles $\left(1-10^{\circ}\right)$, but becomes more incoherent at higher scattering angles [102]. Elastically scattered electrons are mainly exploited in conventional TEM and diffraction methods [101]. If the electron loses energy due to the interaction with the sample, the scattering is said to be inelastic. The scattering angle is small $\left(<1^{\circ}\right)$ and inelastically scattered electrons are almost always incoherent [102]. Energy transfer to the specimen results in the generation of numerous signals. Characteristic X-rays and Auger electrons are emitted due to inner-shell ionization of atoms, secondary electrons are ejected from the valence or conduction bands, deceleration of electrons gives rise to Bremsstrahlung X-rays, and electron-hole pairs may be generated (cathodoluminescence), leading to recombination and emittance of photons in the visible range. These signals are utilized in analytical TEM [101].

Another advantage of using electrons for imaging is that the achievable spatial resolution in TEM is far superior to that of a visible-light microscope. The classic Rayleigh criterion states that the smallest distance that can be resolved is directly proportional to the wavelength of the radiation used for imaging. Thus TEM benefits greatly from the small electron wavelength at high acceleration voltages (e.g. $2.51 \mathrm{pm}$ at $200 \mathrm{kV}$, see equation (15)). It has to be noted though that the ultimate resolution of TEM is limited by instrumentation stability, lens defects and specimen thickness [102].

At this point it also has to be mentioned that while TEM certainly is one of the most versatile and useful characterization instruments in materials science, there are several limitations to the technique. These include the difficult, timeconsuming, and destructive preparation of thin, electron-transparent specimens, the small sampling volume, often difficult image interpretation, and possible electron beam damage to the specimen material [102].

\subsection{Basic Operating Principles}

In the present study, a FEI Tecnai $\mathrm{G}^{2} \mathrm{TF} 20$ UT TEM operated at $200 \mathrm{kV}$ in high vacuum conditions was used for sample characterization. It is equipped with a field emission gun as an electron source, which produces an electron beam of higher brightness and spatial coherence compared to thermionic sources.

The following paragraphs give a very simplified description of the basic operating principles in a TEM: A TEM can be divided into the illumination and the imaging system as illustrated in Fig. 11. The illumination system (Fig. 11(a)) extends from the electron gun to the sample and hosts the condenser lenses one (C1) and two (C2). C2 can be underfocused to form a (nominally) parallel beam in the conventional TEM mode or focused to form a convergent beam in scanning (STEM) mode. A small C2 aperture can be used to create a more parallel 
beam in TEM mode, but cuts off electrons, leading to a reduced probe current at the specimen. Similarly, C1 can be overfocused, resulting in a smaller electron probe in STEM mode and fewer electrons reaching the specimen. In general, the condenser lens and aperture system controls the intensity, coherency, convergence, current, and centering of the electron beam [102].

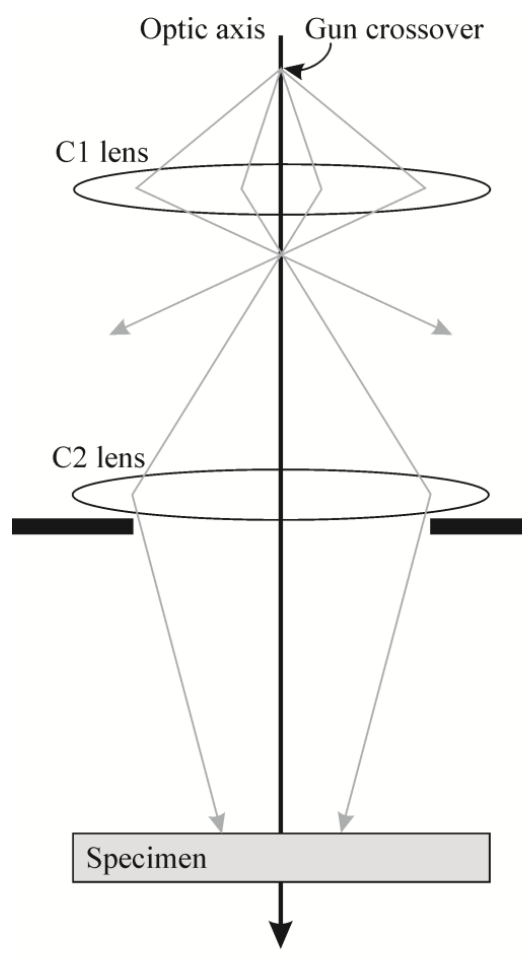

(a) Illumination system

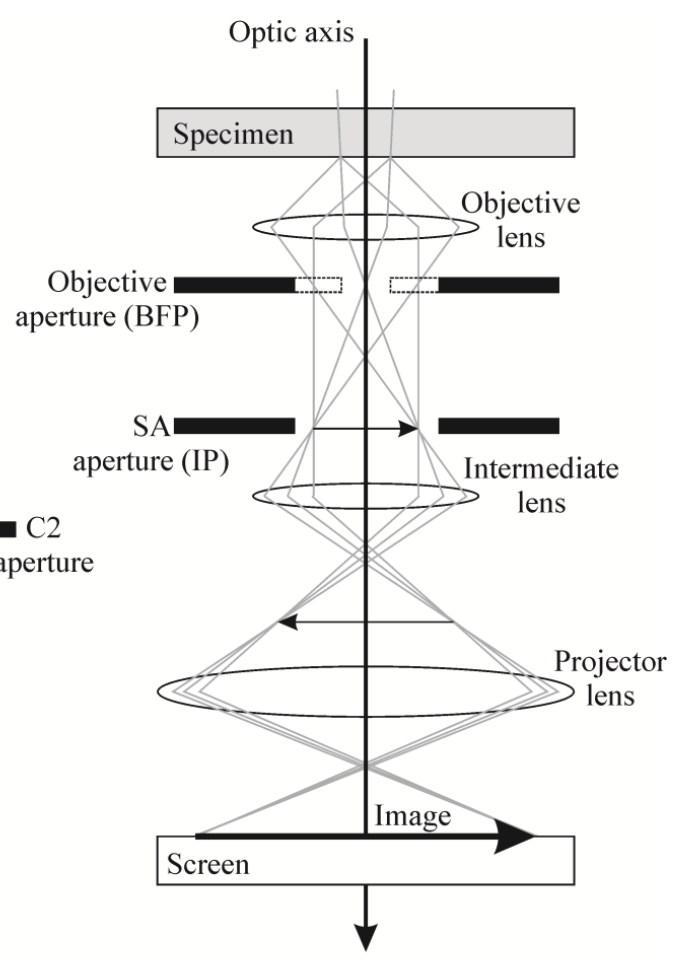

(b) Imaging system

Figure 11: Simplified outline of a TEM consisting of the (a) illumination and (b) imaging system. The illumination system in (a) is depicted in conventional TEM mode, with an underfocused $\mathrm{C} 2$ lens forming a nominally parallel electron beam at the specimen. The imaging system in (b) is shown in imaging mode (note that the SA aperture would normally be retracted in imaging mode). A small objective aperture as indicated by the dashed rectangles can be used to block scattered electrons and thus form a bright-field image.

The imaging system covers the TEM column from the specimen to the fluorescent viewing screen (Fig. 11(b)). It contains the objective lens and aperture, the selected area (SA) aperture, an intermediate lens, and the projector lens. The objective lens collects the electrons emerging from the specimen and disperses them to form a diffraction pattern in the back-focal plane (BFP), then recombines them to create an image in the image plane (IP). By adjusting the intermediate lens so that its object plane coincides with the BFP or IP of the objective lens, 
either the diffraction pattern or the image is projected onto the viewing screen. In imaging mode the objective aperture is inserted into the BFP of the objective lens to exclude scattered electrons from image formation (indicated by the dashed rectangles in Fig. 11(b) and discussed in more depth in chapter 5.2.1). In diffraction mode the SA aperture is inserted into the IP of the objective lens to select a specific area of the specimen to contribute to the diffraction pattern and thus create a selected area electron diffraction pattern (SADP) [102].

\subsubsection{Bright-field and dark-field imaging}

In TEM mode, the objective aperture is used to select either the direct beam or a diffracted beam from a SADP. An image formed by the direct electron beam is called bright-field image and contrast formation is governed by the weakening of the intensity of the direct beam due to interaction with the specimen. An image formed by one or more diffracted beams is known as dark-field image. Only crystals oriented according to the chosen diffracted beams will appear in the dark-field image [102].

In STEM mode, the direct and diffracted beams are not selected by an aperture, but by an on-axis bright-field detector or an off-axis annular dark-field detector, respectively. This is depicted schematically in Fig. 12(a) [102].

\subsection{Imaging Techniques}

Contrast formation in a TEM occurs due to changes in the amplitude or phase of the electron wave as it passes through the specimen. In most practical cases there is a contribution of amplitude and phase contrast, but for easier image interpretation conditions where one mechanism dominates over the other should be chosen [102].

\subsubsection{Mass-thickness contrast}

Mass-thickness contrast is a type of amplitude contrast and is the most "intuitive" way of contrast formation in a TEM. Due to the Coulomb interaction with specimen atoms, electrons are scattered from their direct path through the sample. Heavier elements are more powerful scattering centers, since they carry a higher number of charges and are thus more likely to deflect an electron. Similarly, in thicker samples the likelihood of a scattering event is increased because of the higher number of specimen atoms in the path of the electron. Therefore, in a bright-field image thicker/higher-mass regions of the sample will appear darker than thinner/lower-mass areas. The opposite applies for dark-field images. Massthickness contrast generally dominates at lower magnifications [101,102]. 


\subsubsection{Diffraction (Bragg) contrast}

Diffraction contrast is a form of amplitude contrast in which coherent elastic electron scattering occurs at discrete Bragg angles. In a crystalline specimen atoms are arranged periodically. When these atoms act as scattering centers, constructive or destructive interference of the scattered electrons occurs and discrete diffracted electron beams are generated. The condition for constructive interference is given by Bragg's law, which treats the diffraction as a reflection of the electron waves at the lattice planes.

$$
n \lambda=2 d \sin \theta_{B}
$$

Here, $n$ is an integer, $\lambda$ the electron wavelength, $d$ the lattice spacing in the crystalline specimen, and $\theta_{B}$ the Bragg angle. Moreover, the structure factor of the investigated material has to be considered, which accounts for atomic positions in the unit cell, and can modulate or completely cancel some reflections in the diffraction pattern $[8,101,102]$.

Strong TEM diffraction contrast can be achieved under so-called two-beam conditions, where in addition to the direct beam only one diffracted beam is strongly excited by tilting the specimen. As described in chapter 5.2.1 the objective aperture is used to select either the direct or diffracted beam to form a bright-field or dark-field image, respectively. Electrons contained in the strongly excited second beam have been diffracted by planes fulfilling the Bragg condition. Thus a darkfield image now provides specific orientation information contrary to the general scattering information obtained by mass-thickness contrast [102].

\subsubsection{Z-contrast}

Atomic number $(Z)$ contrast imaging is an extension of mass-thickness imaging. In this STEM technique a high-angle annular dark-field (HAADF) detector is used to gather electrons scattered at angles larger than $50 \mathrm{mrad}$, where there are no more contributions from Bragg diffracted electrons. The HAADF detector can be shifted to high scattering angles by decreasing the camera length as indicated in Fig. 12(a). Heavy atoms with high atomic numbers are more likely to scatter electrons to such high angles, so this technique can be used to obtain elemental contrast. Sample regions containing heavier atoms will appear brighter in the resulting dark-field image (Fig. 12(b)) [8,101,102]. A further advantage of HAADF imaging is that the contrast is usually insensitive to small changes in defocus or specimen thickness. It is also possible to achieve atomic resolution in Z-contrast images [102-104].

An example of the suitability of $Z$-contrast imaging in diffusion barrier assessment is presented in Paper I, where a $\mathrm{Cu}$ diffusion zone is identified by contrast variation in a HAADF image [9]. 


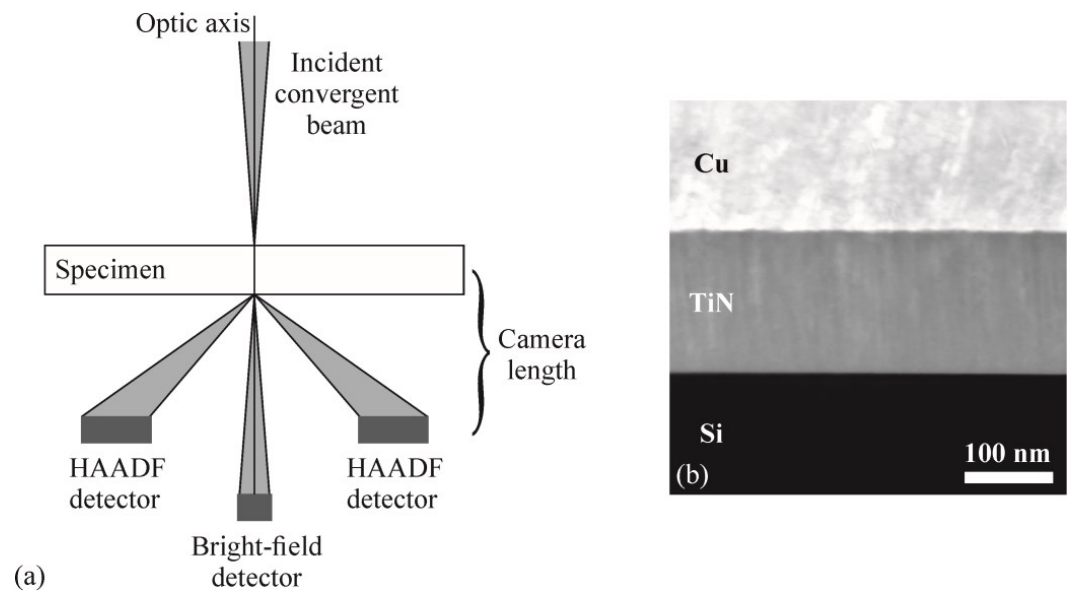

Figure 12: The off-axis HAADF detector setup for $Z$-contrast imaging is illustrated in (a). A cross-sectional $Z$-contrast image of a TiN/Cu stack grown on Si obtained with a HAADF detector is shown in (b). The materials appear progressively brighter going from the low-mass $\mathrm{Si}$ substrate to the high-mass $\mathrm{Cu}$ toplayer. The camera length was chosen sufficiently small for the HAADF detector to only gather electrons scattered at angles larger than $50 \mathrm{mrad}$, so that only minimal diffraction contrast is observable. Thus, the polycrystalline TiN layer is imaged almost featureless.

\subsubsection{Phase contrast (HRTEM)}

In this chapter the terms electron beam and wave will be used interchangeably when appropriate and both actually refer to the electron wave function. The mathematical treatment of phase contrast formation is beyond the scope of this thesis.

Phase contrast is a very sensitive imaging technique, because it is influenced by many factors such as changes in thickness, orientation, and scattering factor of the sample as well as focus and aberrations of the objective lens. This can make phase contrast images difficult to interpret, but on the other hand it can be used to investigate the atomic structure of materials. The imaging techniques described in the previous chapters all require the selection of specific beams to form an image. To acquire a phase contrast image, more than one beam must be selected and in general the image resolution increases with the number of beams contributing to phase contrast formation. The direct and diffracted electron waves experience a relative phase shift after interacting with the atomic potentials of the sample atoms, depending on the strength and spacing of the periodic lattice potential. Thus, an image related directly to the structural periodicity of the crystalline specimen (but not necessarily to the real position of an atom!) is formed in the IP, where the direct and diffracted beams interfere again $[8,102]$. 
However, TEM is always more complicated at a second glance than it appears at first. To interpret a phase contrast image, a "linear" relationship between the image and the sample structure is assumed. This assumption is only valid in the case of a thin specimen that ensures kinematical scattering and linear interference conditions are met. Kinematical scattering means that an electron only undergoes one scattering event within the specimen, while linear interference only considers interference of the diffracted beams with the direct beam and not with each other. This is known as the weak phase object approximation [102].

It also has to be considered that the electron wave is not only phase shifted due to interaction with the crystal potential, but also by a microscope phase factor. A phase contrast transfer function (CTF) is used to describe how aberrations and other system artifacts in a TEM modify the phase contrast image of a specimen. The CTF is the product of a sinusoidal aberration function $\sin \chi(q)$, which depends on the defocus and spherical aberration of the objective lens, an envelope function due to finite temporal coherence $E_{t}(q)$ because of chromatic aberration, focal and energy spread, and instabilities in high tension and objective lens current, and an envelope function due to finite spatial coherence $E_{s}(q)$ because of beam convergence $[102,105]$.

$$
\mathrm{CTF}=\sin \chi(q) E_{t}(q) E_{s}(q)
$$

where $q$ is the spatial frequency (reciprocal lattice spacing). A representative CTF for the FEI Tecnai $\mathrm{G}^{2}$ TF 20 UT TEM used within this study is plotted in Fig. 13 [106]. The point resolution of the microscope is defined as the inverse spatial frequency where the CTF first becomes zero. This utilized TEM can reach a point resolution of $1.9 \AA$. A negative CTF represents positive phase contrast, and atomic columns appear dark on a bright background. Whenever the CTF crosses the $\mathrm{x}$-axis, there is a contrast inversion. Thus, the point resolution is the limit up to which phase contrast images can be interpreted "intuitively" [102].

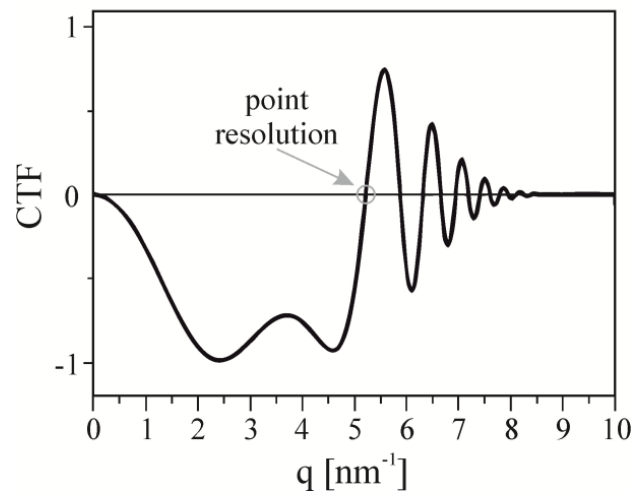

Figure 13: Representative CTF of the utilized FEI Tecnai $\mathrm{G}^{2}$ TF 20 UT TEM adapted from [106]. The point resolution is $1.9 \AA$. 
For best phase contrast imaging conditions the CTF should be close to -1 (or +1$)$ as long as possible before it crosses the abscissa, so that all objects exhibit the same contrast regardless of their reciprocal spacing. Scherzer recognized the possibility of balancing the detrimental effect of spherical aberration against a negative defocus in the aberration function and thereby optimizing the CTF. This is known as the Scherzer defocus $\Delta f_{S c h}[102,107]$.

$$
\Delta f_{S c h}=-1.2 \sqrt{C_{S} \lambda}
$$

with $C_{S}$ as the spherical aberration coefficient and $\lambda$ as the electron wavelength. In practice the Scherzer defocus can be adjusted with the help of automated Fourier transforms generated by the TEM imaging software. These Fourier transforms represent the two-dimensional equivalent to the one-dimensional (squared) aberration function.

In the present study, HRTEM is employed to characterize the atomic structure of the TiN diffusion barriers, especially at the interfaces with the substrate and the $\mathrm{Cu}$ metallization. Several cross-sectional phase contrast images of the interfaces are presented and discussed in Papers I and II. To complete the picture, two HRTEM images of the single-crystal $\mathrm{Cu}$ and TiN films recorded away from the interfacial regions are displayed in Fig. 14. Once again it is stressed that HRTEM

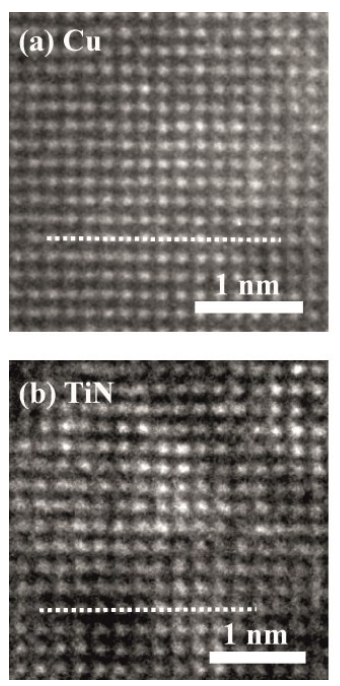

(c) Line intensity profiles

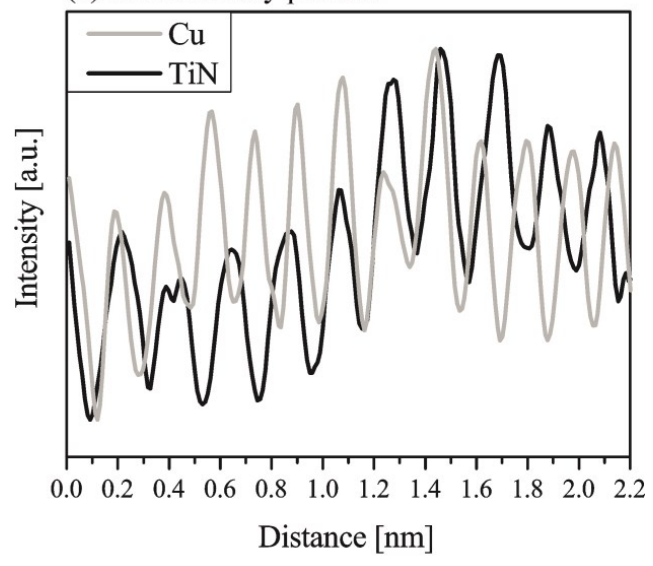

Figure 14: Cross-sectional HRTEM images of the (a) pristine $\mathrm{Cu}$ layer and (b) pristine TiN layer recorded along the [100] zone axis. The white dotted lines mark the regions in both images where the normalized line intensity profiles displayed in (c) have been obtained. The distance of the atomic columns corresponding to the $(020)$ planes is clearly smaller in the case of $\mathrm{Cu}$ $(0.18 \mathrm{~nm})$ than for $\operatorname{TiN}(0.21 \mathrm{~nm})$. 
images generally are not a real representation of the atomic structure of a material and the location of a fringe in the image does not necessarily correspond to the location of a lattice plane. But since the intensity in a phase contrast image varies with a periodicity directly related to the spacing of the lattice planes, HRTEM provides information about lattice spacing and orientation [102]. This is demonstrated in Fig. 14(c) in comparative line intensity profiles extracted for the (020) planes in $\mathrm{Cu}$ and TiN. From these profiles the distance between the atomic columns corresponding to the (020) planes can be determined. The $(010)$ planes are not visible since this reflection is forbidden by the structure factor in fcc materials.

\subsection{Electron Diffraction in the TEM}

Diffraction of electrons in a crystalline specimen proceeds similar to X-ray diffraction, with the difference that electrons interact with both the atomic nuclei and electrons in a material through Coulomb forces and are not reflected by the lattice planes but transmitted through the specimen. As described in chapter 5.2 after interaction with the specimen the objective lens disperses the electrons in the BFP to form a diffraction pattern. This diffraction pattern gives information about the crystallographic characteristics (lattice parameter, symmetry, texture), grain morphology, and orientation of different phases in a sample material [102].

There are two ways of obtaining a diffraction pattern in the TEM. SADPs are formed by inserting a SA aperture in the IP of the objective lens to yield sharply focused spot patterns. These patterns are the basis for all HRTEM investigations, as they allow the specimen to be tilted to a specific zone axis. Alternatively, convergent beam electron diffraction patterns appearing as arrays of discs can be formed, but will not be discussed within this thesis [102].

A single-crystal specimen oriented with a zone axis parallel to the electron beam will give rise to a symmetrical array of diffraction spots, which essentially represent the lattice planes in reciprocal space $[102,108]$. An example is shown in Fig.15(a) in a SADP of a single-crystal TiN/Cu stack recorded at the interface.
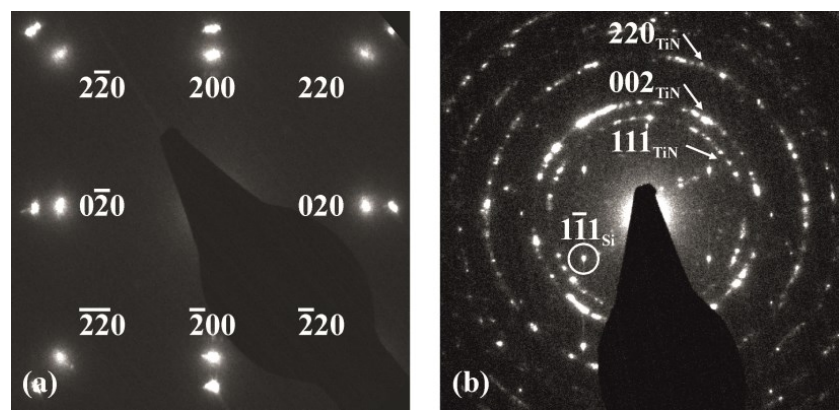

Figure 15: Indexed SADPs of (a) a single-crystal TiN/Cu stack grown on $\mathrm{MgO}(001)$ and (b) a polycrystalline TiN layer grown on $\mathrm{Si}(001)$. 
Indexing of this SADP proceeds as follows: The distance $R$ between the direct and a diffracted beam (scattering angle $2 \theta$ ) is determined by the camera length $L$ as $R=L \tan (2 \theta)$. With Bragg's law from equation (16), $\lambda$ as the electron wavelength, and the assumption that $\theta$ is small, this yields an inverse relationship between $R$ and the lattice spacing $d$.

$$
R d=\lambda L=\text { const }
$$

Furthermore, in cubic materials the spacing $d$ between adjacent (hkl) planes is related to the lattice constant $a$, so that

$$
d_{h k l}=\frac{a}{\sqrt{h^{2}+k^{2}+l^{2}}}
$$

Thus, when the camera constant $\lambda L$ is known (usually by obtaining a SADP of a reference material with known lattice spacings such as $\mathrm{Si}$, see Fig. 15(b)), the diffraction spots can be related to the respective lattice planes in real space.

In case of a polycrystalline specimen that contains crystals of random orientations, the resulting ring diffraction pattern is the sum of all individual spot patterns [102,109]. An example of a polycrystalline TiN layer grown on $\mathrm{Si}$ is shown in Fig. 15(b). The encircled (1111) reflection of Si was used to determine the camera constant. Brighter sections of the diffraction rings in this case are not related to film texture but to the restricted sample area and thus the limited number of grains illuminated within the SA aperture.

\subsection{Analytical TEM}

Analytical TEM encompasses the techniques of energy-dispersive X-ray spectroscopy (EDX), electron energy-loss spectroscopy, and energy-filtered TEM. Only the first will be discussed within this thesis. It is convenient to perform analytical TEM in STEM mode where the beam is already converged to form a probe for analysis [102,110].

When interacting with the specimen, electrons generate two kinds of X-rays. The ejection of a core or inner-shell electron leads to a cascade of electron transitions (denoted as K, L, M, etc.) resulting in a characteristic X-ray (or Auger electron) with a well-defined energy. Bremsstrahlung on the other hand arises due to the deceleration of electrons by electrostatic interaction with the atomic nuclei. Thus, the recorded X-ray spectrum consists of element-specific characteristic peaks on a non-characteristic background. Two such spectra are depicted in Fig. 16. In STEM mode it is possible to obtain EDX spectra from distinct analysis points within the sample, but also elemental profiles or even two-dimensional elemental maps. Examples of the latter and their application to diffusion studies are dis- 


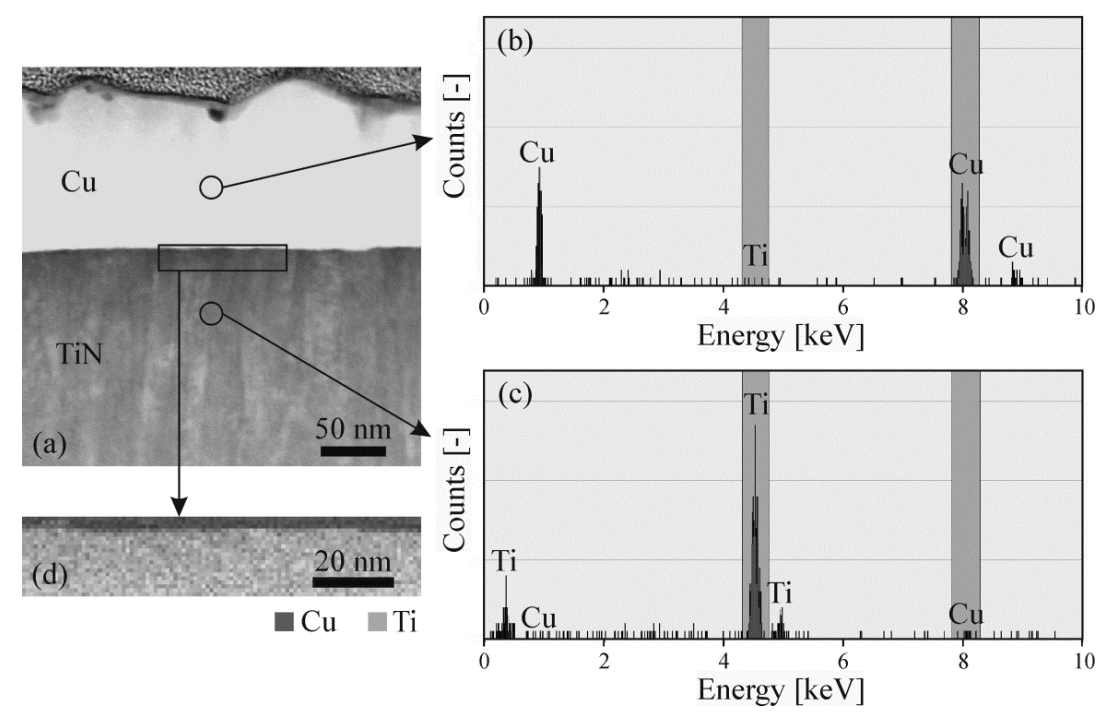

Figure 16: (a) STEM HAADF image of a Cu/polycrystalline TiN interface after annealing the sample at $600{ }^{\circ} \mathrm{C}$ for $1 \mathrm{~h}$. Corresponding EDX spectra were obtained within the (b) $\mathrm{Cu}$ and (c) TiN layer. The $\mathrm{Cu}-\mathrm{K}$ and Ti-K peaks are highlighted in both spectra due to their suitability for subsequent elemental mapping because of high counts and no peak overlap. An elemental map of the $\mathrm{Cu}-\mathrm{K}$ and Ti-K edges recorded across the interface as indicated by the rectangle is shown in (d).

cussed in the appended Papers I and II. An elemental map of the $\mathrm{Cu}-\mathrm{K}$ and the Ti-K edges is also displayed in Fig. 16(d) for a polycrystalline TiN/Cu stack after annealing at $600{ }^{\circ} \mathrm{C}$ for $1 \mathrm{~h}$. No diffusion across the interface is observable.

The spatial resolution of elemental EDX maps is essentially determined by the probe size [110], while the energy resolution is limited to app. $140 \mathrm{eV}$ by the detector [102]. Difficulties when performing EDX analyses in a TEM include low count rates due to the small interaction volume of the thin specimens, sample drift when acquiring maps, and stray radiation from the illumination system, sample holder, or specimen grid. To allow for the latter, mostly Mo grids were employed in the present study instead of the standard $\mathrm{Ti}$ or $\mathrm{Cu}$ grids. Limitations not inherent to the TEM system are peak overlaps in the spectrum and inaccuracies in detecting low-mass elements [102]. 



\section{ATOM PROBE TOMOGRAPHY}

\subsection{Background and Instrumentation}

As Larson et al. put it in their latest book on the subject, APT is "one of the most spectacular microscopies that exist" [111]. It produces a three-dimensional reconstruction of a specimen with single-atom sensitivity. This allows for the determination of elemental concentration profiles in any direction, characterization of interfaces, and the investigation of ordering, dopant interactions, cluster formation, diffusion, and early stages of precipitation. Under certain conditions even information about crystal structure may be gained [111-113].

\subsubsection{From field ion microscopy to atom probe tomography}

APT has its roots in field ion microscopy (FIM), which images specimen surfaces with atomic resolution. The technique is based on the ionization of an inert imaging gas in the vicinity of a positively charged needle-shaped sample. An electric field at the sample surface is generated by a high voltage applied to the metallic specimen, which causes the free electron cloud to be displaced by a small amount, leaving the surface positively charged. Ionized gas atoms are accelerated away from the specimen towards a phosphor screen, where the resulting maps represent the distribution of the electric field at the tip surface. This can be related to the local tip topography. A further increase in voltage eventually results in the field-evaporation of atoms (or, more correctly, ions) from the tip surface. The electric field strength $F$ at the tip apex is directly proportional to the applied voltage $V$ and inversely proportional to the tip radius $R$.

$$
F=\frac{V}{k_{f} R}
$$

Here, $k_{f}$ is a constant that accounts for the tip shape and its electrostatic environment and is called the field factor. In the present study, the standard value of 3.3 was used for $k_{f}$. Since the tip is usually approximated as a truncated cone with a hemispherical cap, the radius of the hemisphere is synonymous with the radius of curvature of the tip. The half angle at the cone apex is termed shank angle. In addition to the needle-shape requirement it is also necessary to cool the specimen to cryogenic temperatures in FIM. Cryogenic temperatures limit the thermal movement and diffusion of tip surface atoms and provide thermal accommodation of the imaging gas particles, which leads to increased spatial resolution [112]. 
Ionized imaging gas atoms or field-evaporated specimen ions are accelerated along the electric field lines away from the tip surface. The easiest model to describe this is a simple point-projection. Assuming the phosphor screen is a distance $L$ away from the tip, the image magnification $M_{p r o j}$ can be expressed as

$$
M_{\text {proj }}=\frac{L}{\xi R},
$$

with $\xi$ as a constant called the image compression factor (ICF). The ICF describes the deflection of the electric field lines and is an instrument parameter. In the employed APT system LEAP 3000X HR it has a value of 1.65. The magnification is typically higher than $10^{6}$, thus enabling to resolve the positions of individual atoms [112].

After being able to look at images of single atoms, the next logical step was to try to identify these atoms with the help of a time-of-flight (TOF) mass spectrometer. To know the start time for a field-evaporated ion, pulsing of the evaporation events is necessary. This can be achieved by superimposing the DC voltage applied to the specimen with high-voltage (HV) pulses. However, this approach limits suitable specimen materials to metals because it requires a high electrical conductivity to accomplish electric field pulsing. To overcome this limitation, instruments employing laser pulsing have been developed. These APTs enable the investigation of materials from the entire spectrum of solids, including semiconductors, ceramics, and organic and biological materials [114]. There are complementary if not conflicting theories as to the exact mechanisms leading to the laser pulse-induced evaporation of surface atoms. The two suggested processes are sublimation assisted by the electric field component of the laser beam and thermally-induced evaporation of atoms from the tip apex [115-117]. Two recent textbooks on the subject favor the second theory [111,112]. All APT investigations reported within this thesis have been performed in laser pulsing mode.

Another leap (no pun intended) in instrumentation came with the development of the local electrode atom probe (LEAP). The close proximity of the counterelectrode to the specimen enhances the electric field and thereby lowers the necessary applied voltage. This ultimately results in higher data collection rates and better mass resolution, as well as an increased field-of-view [111]. A schematic of a LEAP system is shown in Fig. 17.

\subsubsection{Operating principle}

The setup of a LEAP (and an APT in general) comprises an ultra-high vacuum chamber with a base pressure of the order of $10^{-11}$ mbar. The specimen is introduced via several load-lock chambers and eventually mounted on a stage that can be moved in $x$-, $y$-, and $z$-direction. The specimen is then aligned with the local 


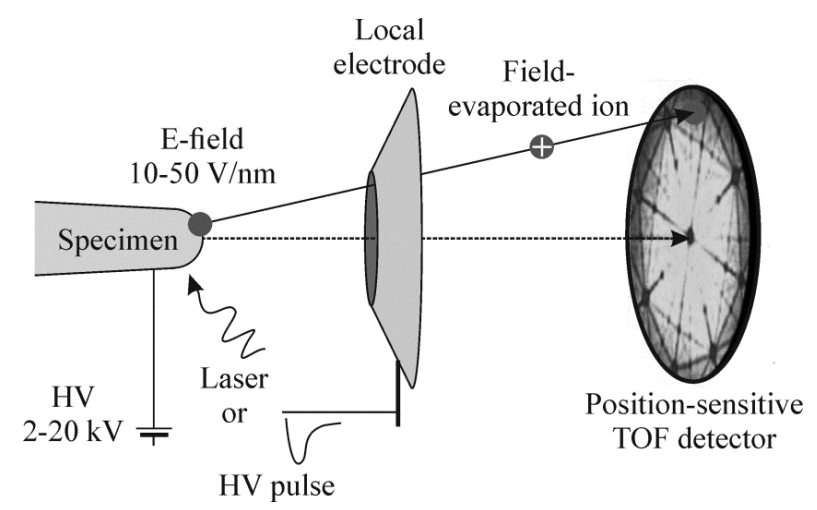

Figure 17: Schematic (not to scale!) of a LEAP setup. An ultra-high vacuum chamber hosts the cryogenically cooled specimen, the local electrode, and a position-sensitive TOF detector. Field-evaporation of ions is induced either by HV- or laser pulsing.

electrode aperture, which has a diameter of $40 \mu \mathrm{m}$. The distance between specimen tip and local electrode is app. $20 \mu \mathrm{m}$, while the distance $L$ between the specspecimen and the detector is $382 \mathrm{~mm}$ in the LEAP system employed for this study (distances not to scale in Fig. 17) [112]. Liquid helium is used to cool the specimen to temperatures as low as $20 \mathrm{~K}$. A standing DC voltage in the range of $2-20 \mathrm{kV}$ is applied to the tip to generate an electric field of $10-50 \mathrm{~V} / \mathrm{nm}$ at the apex. In laser-assisted LEAPs, a laser beam is focused on the specimen tip through a window in the chamber or with the help of in-vacuum optics. The LEAP system used for this work is equipped with a green laser $(\lambda=532 \mathrm{~nm})$, but systems of the latest generation employ ultraviolet lasers. Laser pulses can have sub-ns durations at repetition rates larger than $100 \mathrm{kHz}$. In voltage pulsing mode, the counter-electrode is connected to a high-voltage pulser delivering negative $\mathrm{HV}$ pulses in the ns range. The underlying standing voltage is regulated to achieve a target field-evaporation rate, which in the present study was set to $0.5 \%$. This means that for every 1000 pulses, five ions are field-evaporated $[111,112]$.

The field-evaporated ions are detected by a delay-line detector based on microchannel plate (MCP) technology. The detection efficiency is limited to around $40-50 \%$ by the efficiency of these MCPs, but does not depend on ion energy or mass. These detectors measure the TOF of each ion and record the position of the impact. The TOF is defined as the time between the application of the pulse and the moment the ion hits the detector. It can be directly related to the mass-tocharge ratio $(\mathrm{m} / \mathrm{n})$ of the ion.

$$
\frac{m}{n}=2 e V\left(\frac{T O F}{L}\right)^{2}
$$

Here, $e$ is the elementary charge, $V$ is the total applied voltage, and $L$ is the flight path $(382 \mathrm{~mm})$ [112]. 
A modern LEAP system operated in laser pulsing mode achieves a mass resolving power (MRP, $m / \Delta m$ at FWHM) of 1000 with a field-of-view larger than $150 \mathrm{~nm}$. The analytical sensitivity is as high as $1 \mathrm{ppm}$ with a measurement rate of more than $10^{7}$ atoms per hour. The spatial resolution is better than $0.3 \mathrm{~nm}$ in all directions and volumes on the order of $10^{6} \mathrm{~nm}^{3}$ can be analyzed $[111,114]$.

On the other hand, one of the main limitations of the technique is, similar to TEM, the difficult and time-consuming sample preparation. Furthermore, not all prepared specimens will run successfully in the experiment and some will fracture as soon as the standing voltage is applied. Once the specimen is measured, the $3 \mathrm{D}$ reconstruction might prove problematic, especially in the case of more complex material structures such as thin film stacks, as will be discussed in chapter 6.3 [114].

\subsection{Specimen Preparation}

In analogy to TEM, the specimen can be described as the "primary optic" in APT, as its geometry controls the ion trajectories from the tip surface to the detector. An APT specimen must fulfill three fundamental requirements: (i) the tip must be sharp enough to facilitate field evaporation, (ii) it must mechanically withstand the applied electric field, and (iii) the feature of interest must be included within the first $100 \mathrm{~nm}$ of the specimen apex [111]. Of the two established techniques to produce such specimens, namely electropolishing and focused ion beam (FIB) methods, only the latter was employed within this study and shall be briefly described here.

\subsubsection{Site-specific FIB sample preparation}

The advantage of using an ion beam for the preparation of specimen tips is that the tips can be sharpened and imaged simultaneously by detecting SEs or BSEs inside a scanning electron microscope. This allows for the site-specific preparation of samples, so that particular regions of interest (ROIs) are contained within the sample volume. FIB methods are applicable to almost all materials. Most FIB systems employ a $\mathrm{Ga}^{+}$ion beam for cutting and polishing [111].

In a standard lift-out procedure a platinum layer is deposited on top of the sample to protect the surface and mark the extraction region. Subsequently, FIB cuts are performed at an angle to the sample surface to produce a wedge-shaped lamella as visible in Fig. 18(a). An in-situ micromanipulator is Pt-welded to the sample wedge, and used to transfer the lamella to a pre-fabricated array of Si-posts. These Si-posts are also commonly referred to as microtips or needlepad. One end of the lamella is Pt-welded to one of the Si-posts and subsequently cut free to repeat the process on further microtips. The Si-posts have a height of app. $100 \mu \mathrm{m}$ and are placed $450 \mu \mathrm{m}$ apart from each other (Fig. 18(b)). This allows for independent LEAP measurements of neighboring specimen tips [111]. 
A series of annular milling steps is carried out to give the specimen the desired needle shape as depicted in Fig. 18(c). In the present study, a final polishing step with $5 \mathrm{keV} \mathrm{Ga}^{+}$ions was included to remove surface regions damaged due to $\mathrm{Ga}$ implantation.

The described lift-out procedure is known as top-down or normal orientation liftout and was employed to produce the specimens in this study. An alternative is the cross-sectional lift-out, where the interface is faced edge-on. This increases the interface volume contained within the tip and also reduces the stress acting on the interface during analysis [111]. However, this lift-out technique proved unsuccessful in the case of the studied TiN/Cu layer stacks, since the FIB milling rate of $\mathrm{Cu}$ was much higher than for TiN, making it impossible to produce tips with reasonable geometry. Furthermore, the spatial resolution in APT in the analysis- (z-) direction is higher than in the two lateral directions due to ion trajectory aberrations. Thus, the highest spatial resolution for planar interfaces can be achieved with the layers in top-down orientation (normal to the analysis direction) [118].
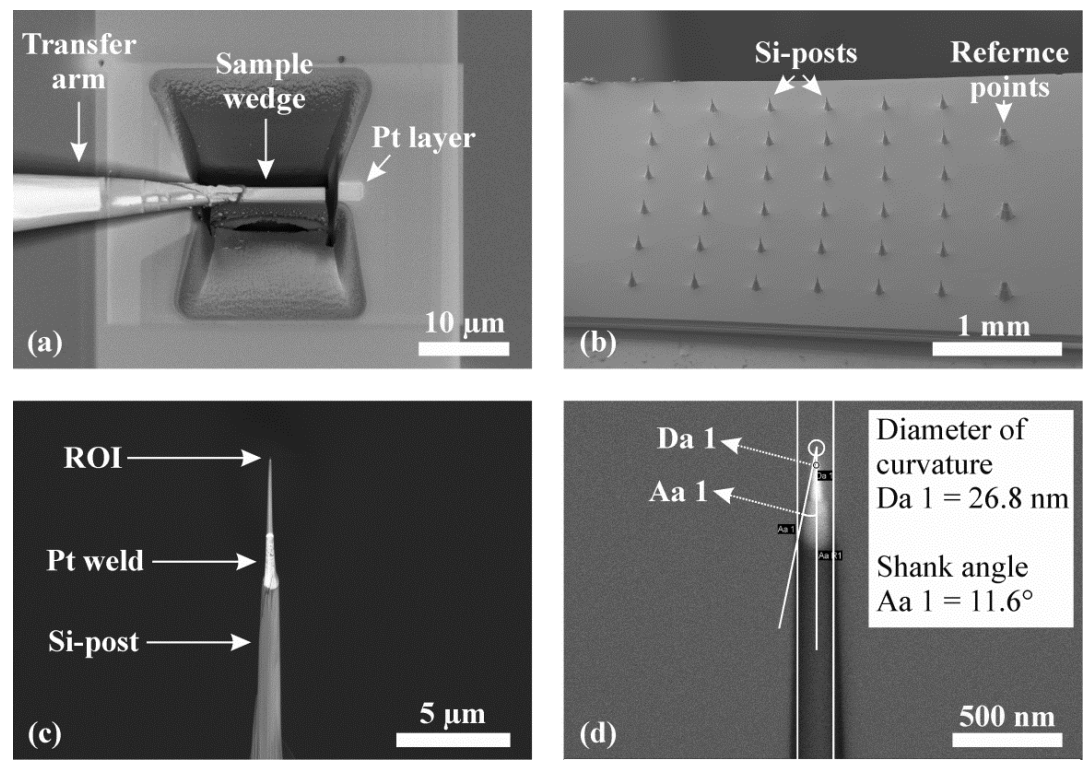

Figure 18: Scanning electron micrographs of (a) the sample wedge being removed with the micromanipulator (plan-view), (b) the needlepad holding 36 pre-fabricated Si-posts, (c) the sharpened specimen welded to a Si-post, and (d) the tip geometry measured in a highmagnification image yielding input parameters for 3D reconstruction. 


\subsection{Data Collection and Reconstruction}

\subsubsection{Measurement parameters}

When measuring an unfamiliar material in a LEAP it is advisable to carry out a parameter study to determine the best possible analysis conditions. Recorded mass spectra should be optimized in terms of MRP and background signals, which are ion evaporation events that occur out of sync with the evaporation pulses. For samples investigated within this study the MRP $m / \Delta m$ at FWHM was around 700 (the system specification is 1000), while the background signal was kept below $20 \mathrm{ppm} / \mathrm{ns}$ at the chosen measurement conditions. Parameters that can be tuned are [111]:

- Pulse rate: The pulse rate should be chosen as high as possible to facilitate a high acquisition rate, but still low enough in order for ions with a long TOF to reach the detector within the pulse. Another issue is insufficient cooling of specimens with poor thermal conductivity during high laser pulse rates. This can result in delayed evaporation of ions which manifests in long thermal tails in the mass spectrum.

- Laser pulse energy: A high laser pulse energy lowers the required evaporation field and generally results in spectra with high MRP and low background. However, it might be detrimental to spatial resolution as it can lead to nonuniform spatial evaporation from the tip surface. Moreover, literature reports suggest that low laser energies are required to achieve the best results when measuring oxides and nitrides $[111,119,120]$. A similar trend is observed in the present study.

- Base temperature: A low base temperature (20-60 K) minimizes surface diffusion and results in better heat transport away from the tip apex due to the higher temperature gradient. Thus, low base temperatures provide the best data quality, and should be used if the specimen can withstand the mechanical stress.

The measurement parameters used for the investigations in this thesis are chosen to optimize the spectral quality, mainly by minimizing thermal tails in the spectra and maximizing the signal-to-noise ratio. They are given in the experimental sections in Papers I and II.

\subsubsection{Data processing and 3D reconstruction}

Data reconstruction proceeds through an algorithm that relates the detector hit sequence and position of ions back to their $(x, y, z)$-position on the specimen tip surface. It is accomplished with the Cameca image visualization and analysis software package IVAS based on an algorithm developed by Bas et al. [121] and later advanced for a larger field-of-view by Geiser et al. [122]. 

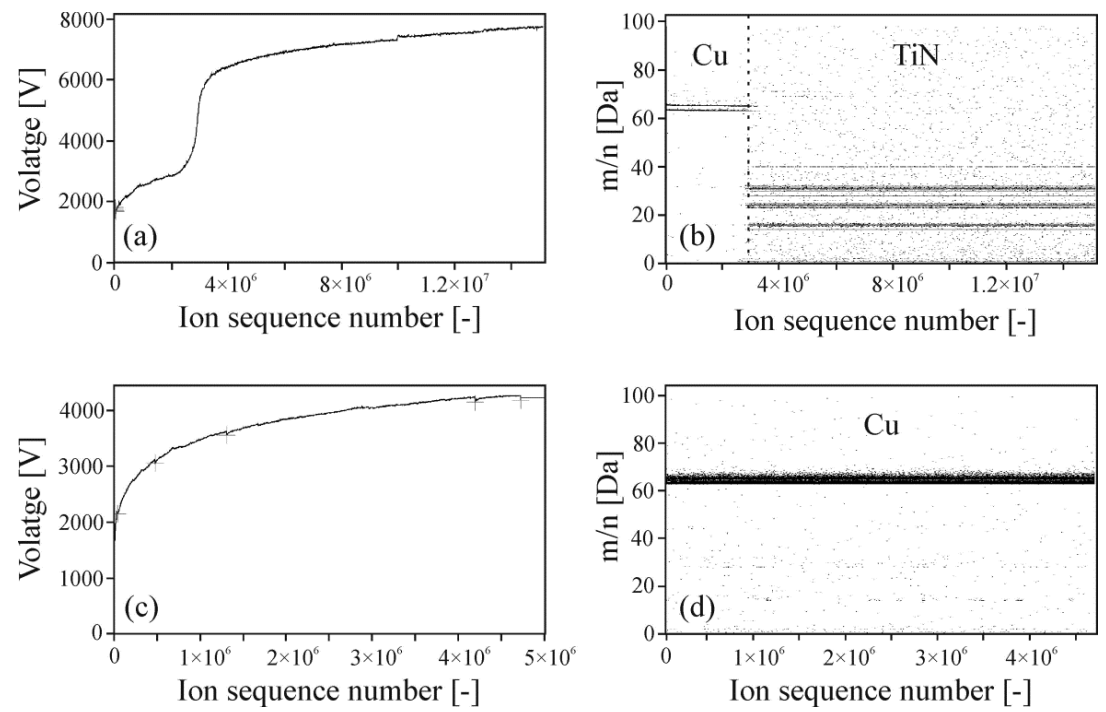

Figure 19: Evolution of evaporation voltage with ion sequence number (left) and corresponding mass-to-charge ration $m / n$ of the detected ions (right) shown for $(\mathrm{a}, \mathrm{b})$ a specimen containing a $\mathrm{TiN} / \mathrm{Cu}$ interface and $(\mathrm{c}, \mathrm{d})$ a specimen prepared from a single $\mathrm{Cu}$ layer.

During a LEAP measurement, the standing voltage is continuously and automatically increased to maintain a constant evaporation rate. The voltage evolution over ion sequence number (translating to the $z$-coordinate) is shown in Fig. 19 for a specimen containing the TiN/Cu interface (Fig. 19(a)) and for a specimen cut only from a Cu layer (Fig. 19(c)). While the evaporation voltage increases steadily in the latter case, a discontinuity is observed when analyzing the tip containing the interface. Indeed, a comparison with the recorded $m / n$ data (Fig. 19(b)) shows that the discontinuity occurs at the transition from the $\mathrm{Cu}$ to the TiN film. This can be related to the higher evaporation field of TiN compared to $\mathrm{Cu}$. Problems arising due to this sudden increase in evaporation voltage will be addressed when discussing 3D reconstruction later in this chapter.

Since APT is a combination of projection imaging and TOF mass spectrometry, a vital part of data processing is the refinement and evaluation of recorded mass spectra. Automated corrections to collected TOF datasets can be executed within the analysis software to account for oversimplified electrostatic assumptions, linear detector response and detector bias ("voltage corrections"), as well as curved TOF isosurfaces ("bowl corrections"). The corrected TOF data is then converted to $m / n$ (see equation 23) [111]. An exemplary cumulative mass spectrum for a TiN/Cu stack is shown in Fig. 20(a). For a successful chemical analysis all observed peaks must be assigned to an ion type according to its isotopic distribution. This task can be complicated by peak overlaps, deviations from natural isotope abundances, and the appearance of molecular ions. To illustrate some of these difficulties a zoom to the $m / n$ region from 59 to $68 \mathrm{Da}$ is provided 
in Fig. 20(b). The two most prominent peaks are related to the two $\mathrm{Cu}^{+}$isotopes at 63 and $65 \mathrm{Da}$ (note that the symbols in Fig. 20(b) do not represent expected peak intensities). However, these peaks overlap with peaks related to the molecular $\mathrm{TiN}^{+}, \mathrm{TiO}^{+}$, and $\mathrm{CuH}_{2}^{+}$ions. In the present case, the consequences of this overlap for the final reconstruction are not severe, since the $\mathrm{Cu}^{+}$peaks are significantly more intense than the others. Still, for a quantitative chemical analysis a decomposition of overlapping peaks has to be performed. The IVAS software package can calculate unknown ion fractions based on information of nonoverlapping peaks and expected isotope abundances. In Paper I the result of such a calculation is compared to compositional information obtained by elastic recoil detection analysis and both are found to be in excellent agreement [9]. On the other hand, for material systems exhibiting significant major peak overlaps or thermal tails, a more advanced algorithm such as the blind peak deconvolution proposed by Johnson et al. has to be employed, which also accounts for system inherent peak broadening [123].
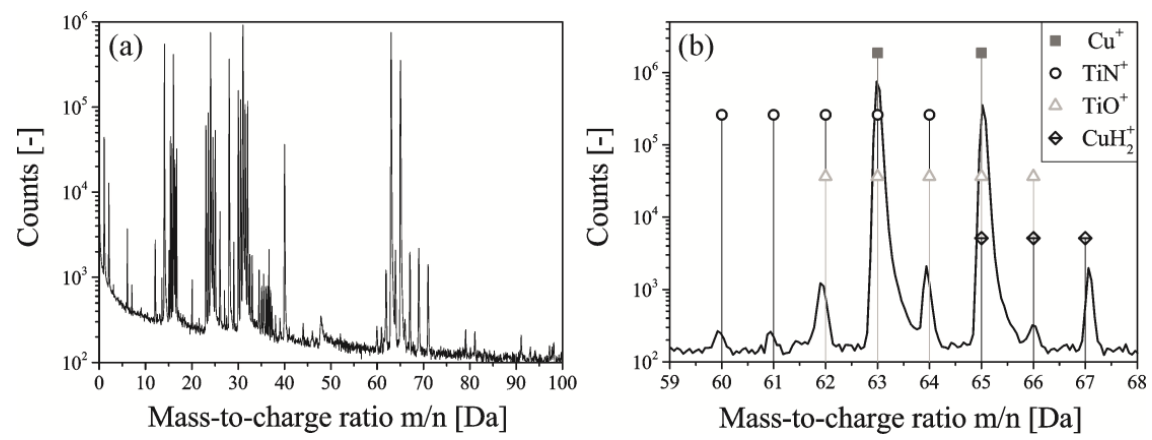

Figure 20: (a) Cumulative mass spectrum of a TiN/Cu stack and (b) zoom illustrating the peak overlaps of the two $\mathrm{Cu}^{+}$isotopes with molecular ions. The symbols in (b) do not represent the relative peak intensities.

Spatial reconstruction of the acquired data is based on magnifying detector hit positions to real-space $x, y$-coordinates (see equation 22), while the $z$-component is determined from the ion number sequence, the assigned ionic volume and the estimated imaged area of the tip surface. The radius evolution of an analyzed tip can be modelled with two different approaches [111]:

- Shank evolution: The increase of the radius during evaporation from the tip is based on geometrical parameters measured prior to the APT investigation (see Fig. 18(d)). A specimen reconstructed based on the shank evolution is shown in Fig. 21(a).

- Voltage evolution: The voltage evolution reconstruction protocol is based on equation 21 , so that the radius increases with the applied voltage. However, if the evaporation field is not constant over the analysis direction, leading to a 
discontinuity in the voltage curve as shown in Fig. 19(a), this results in a distorted reconstruction. In the case of a low-field material on a high-field mamaterial (e.g. $\mathrm{Cu}$ on TiN), the ensuing tip profile will look as depicted in Fig. 21(b).

(a)

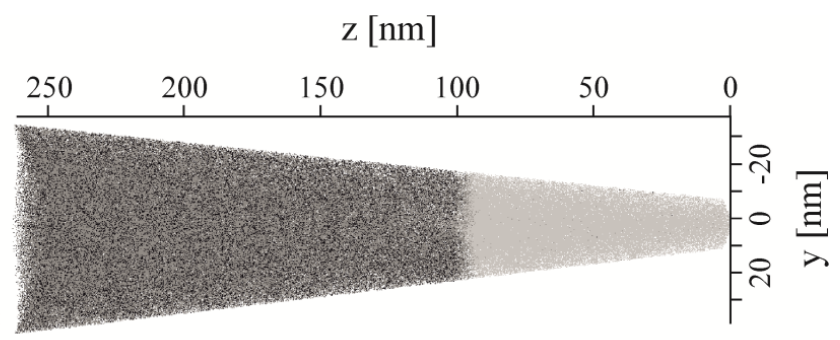

(b)

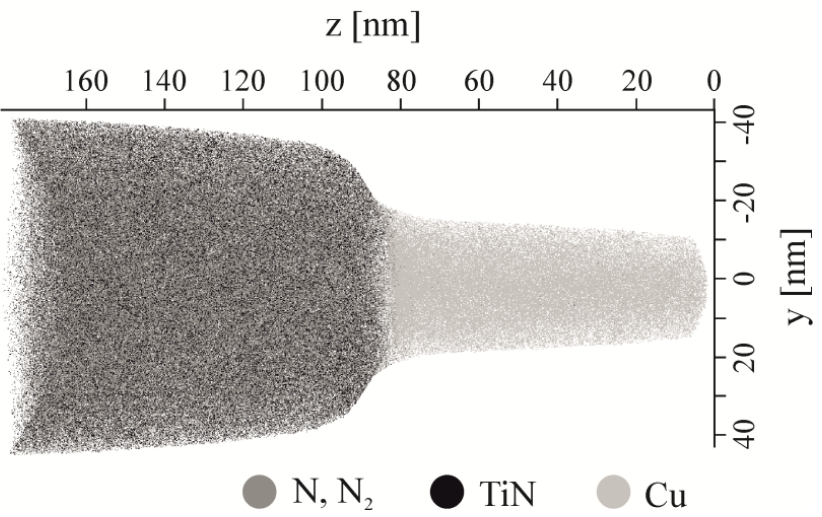

Figure 21: 3D reconstructions of a single-crystal TiN/Cu bilayer specimen in (a) shank evolution and (b) voltage evolution mode. Each plotted data point represents one (molecular) ion, but only a fraction of all measured ions is plotted for better visualization.

Currently, the IVAS reconstruction protocol cannot account for changes in evaporation field within the measurement, making the characterization of thin film stacks a challenging task [124-126]. Investigated specimens in the present study were reconstructed in shank and voltage evolution mode, and both $\mathrm{Cu}$ and $\mathrm{Ti}$ were tested as primary elements determining the evaporation field. All reconstructions were evaluated in terms of interface flatness and relation to the tip geometry observed in the scanning electron microscope. Here, tips that did not fracture before the measurement reached the substrate material proved especially useful. Although the substrate material was always left out of the final reconstruction to avoid peak overlaps and thermal tails, preliminary reconstructions containing the substrate material were used to measure the TiN film thickness in addition to the $\mathrm{Cu}$ toplayer thickness. These layer thicknesses can be used to monitor the conformity of reconstructions and electron micrographs. Somewhat surprisingly, reconstructions based on voltage evolution often yielded better re- 
sults than their shank counterparts in terms of interface flatness and layer thickness. It can be assumed that the shank reconstruction "sugarcoats" the observed tip geometry, which in reality often is not as perfect, for example due to higher milling rates of $\mathrm{Cu}$ compared to $\mathrm{TiN}$ in the FIB instrument. Since it also has been found that in the analyses of multilayer structures the highest spatial resolution is observed particularly near the center of the reconstruction $(x, y=0)$ [125], ROIs defined in the IVAS software package that limit the analyzed sample volume to that region can improve results as well. To ensure consistency, all reconstructions presented in Papers I and II were obtained in voltage evolution mode, with $\mathrm{Cu}$ as the element determining the evaporation field.

\subsection{Application to Diffusion Studies}

High spatial resolution and superior chemical sensitivity make APT a valuable tool for the investigation of diffusion phenomena in thin film systems. For example, laser-assisted APT has been used to gain insights into the spinodal decomposition of TiAlN coatings, which is an uphill diffusion process described by a negative diffusion coefficient [127-129]. It has also been employed successfully to study the distribution and segregation of dopants in materials for microelectronics. A comprehensive review on the subject is available by Kelly et al. [130]. This chapter will present two examples of how diffusion in the Cu metallization-TiN barrier system has been explored and visualized by APT within the present study.

\subsubsection{Chemical depth profiling}

An obvious way to characterize diffusion from a 3D APT reconstruction is to obtain a $1 \mathrm{D}$ chemical depth profile across an interface. This interface does not necessarily have to be oriented normal to the analysis direction, since the IVAS software package allows for the calculation of profiles along any direction through the dataset. Moreover, profiles can be calculated for single ions, decomposed ions or combinations of ions. Fig. 22 shows a comparison of $\mathrm{Cu}$ concentration profiles in single-crystal and polycrystalline TiN diffusion barrier layers before and after annealing treatments. The profiles are acquired across the interfaces 20-30 $\mathrm{nm}$ into the respective TiN barrier layers. The interface is defined at a $\mathrm{Cu}$ concentration of 50 at.\% and the profiles are calculated with a fixed bin width of $0.1 \mathrm{~nm}$. The results are similar to the diagrams presented in Paper I, although these were obtained as proximity histograms from isoconcentration surfaces which shall be discussed in the next subchapter. It is apparent from Fig. 22 that $\mathrm{Cu}$ diffusion into the single-crystal layer can only be observed after the $12 \mathrm{~h}$ annealing treatment at $1000{ }^{\circ} \mathrm{C}$, while the diffusion depth in the case of the polycrystalline barrier is already app. $30 \mathrm{~nm}$ after annealing at $900{ }^{\circ} \mathrm{C}$ for $1 \mathrm{~h}$. This is justified by the different mechanisms of lattice and grain boundary diffusion. 


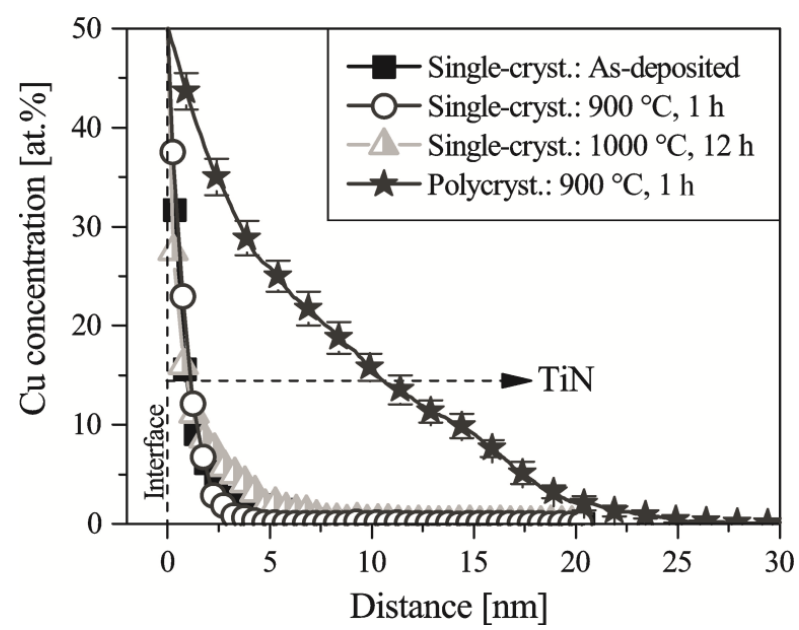

Figure 22: 1D $\mathrm{Cu}$ concentration profiles with error bars in single-crystal and polycrystalline TiN diffusion barrier layers before and after annealing treatments. Not every measured data point is represented by a symbol in the plots. The error bars in the single-crystal cases are smaller than the symbols and therefore not visible.

\subsubsection{Isoconcentration surfaces}

Isoconcentration surfaces can be constructed within a dataset by defining a specific threshold value. This can be an atomic or (molecular) ionic concentration or even a concentration ratio. Instead of obtaining a 1D profile along an explicit direction, so-called proximity histograms can be calculated with respect to distance from the isoconcentration surface. This approach restricts the influence of the interface roughness on the acquired diffusion profile over the interface [111]. Therefore, it is useful when studying the uniform lattice diffusion in singlecrystal TiN barriers. Another conceivable application would be the investigation of type B diffusion regimes (see chapter 2.3.2) in polycrystalline materials, where isoconcentration surfaces could potentially be used to examine the diffusion occurring from the grain boundaries into the grain. However, this has not been in the scope of this thesis.

Exemplary isoconcentration surfaces illustrating $\mathrm{Cu}$ diffusion in single- and polycrystalline TiN barriers after an annealing treatment at $900{ }^{\circ} \mathrm{C}$ are depicted in Fig. 23. In the latter case, non-uniform diffusion of $\mathrm{Cu}$ presumably occurring along a grain boundary is observable (Fig. 23(b)). The volume concentration plots used to demonstrate this in Paper II are an extension of the isoconcentration surfaces. 

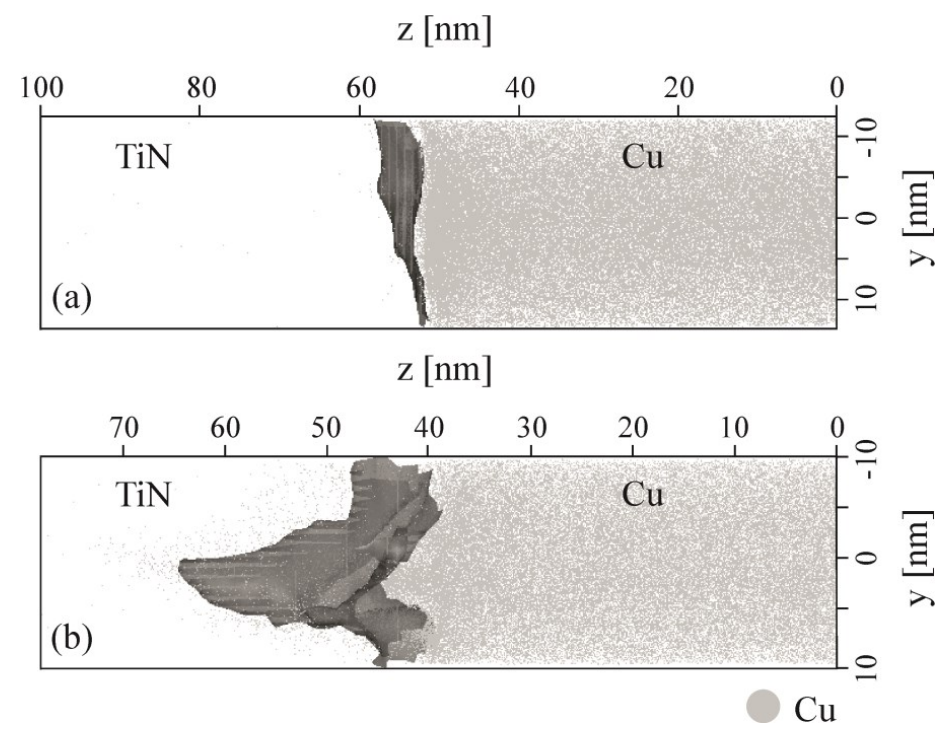

Figure 23: Side-view of cylindrical ROIs containing isoconcentration surfaces plotted at 50 at.\% $\mathrm{Cu}$ for a (a) single-crystal and (b) polycrystalline TiN diffusion barrier layer after a $1 \mathrm{~h}$ annealing treatment at $900^{\circ} \mathrm{C}$. Ions related to the TiN film are not plotted for better clarity. 


\section{CONCLUSIONS AND OUTLOOK}

Within this thesis, diffusion characteristics in the technologically important TiN/Cu thin film system have been investigated by HRTEM and APT. Results presented in Papers I and II allow to draw two main conclusions: Firstly, the microstructure of diffusion barrier layers is a determining factor influencing the barrier performance and in extension the lifetime and reliability of microelectronic devices. While it is as yet not feasible to fabricate single-crystal barrier layers on an industrial level, this study shows that also the efficiency of the frequently employed polycrystalline TiN barriers can be enhanced by limiting the fraction of open, porous grain boundaries within the columnar film. This was achieved by depositing the barrier layers at high substrate temperatures under moderate ion bombardment. A promising approach for future work is to adopt a lowtemperature deposition process shown to produce dense nitride films such as the hybrid DC/high power impulse magnetron sputtering process developed by Greczynski et al. [131] to the needs of microchip manufacturers. Comparisons with first-principles calculations also point towards the possibility of influencing diffusion characteristics of $\mathrm{Cu}$ in TiN by tuning the TiN stoichiometry, although the large-scale technological implementation of this might prove challenging. Moreover, this approach is only viable if preferential diffusion paths such as grain boundaries are eliminated.

From a characterization point-of-view, this thesis demonstrates that the recently emerged APT technique can be used to determine diffusion processes in thin film stacks. However, while APT provides chemical identification on the atomic lev$\mathrm{el}$, only limited structural information can be gained. Therefore, it is important to correlate APT with a more versatile microscopy technique such as (HR)TEM. The combination of the two methods is not only necessary to characterize the TiN/Cu bilayers comprehensively, but can also be used to monitor the agreement between micrographs of the layers and the $3 \mathrm{D}$ reconstruction of the APT specimen tips. Especially when investigating interfaces by APT, the limitations of the reconstruction algorithm with respect to sudden changes in the evaporation fields of layered material systems have to be kept in mind. The "ultimate" microscopy technique would be a hybrid TEM/APT system, able to determine the position, identity, and chemical nature of every atom in a material volume [104]. First steps in this direction are now being made by investigating APT tips before and after evaporation in a TEM instead of preparing separate samples for APT and TEM. However, this approach is still in its infancy, due in large parts to the delicate sample handling required and the fact that state-of-the-art APTs and TEMs are rarely available in the same laboratory. When the technique is more refined in a few years' time and also put into context with numerical models and simula- 
tions, it might well be the solution to the metrology needs projected by the International Technology Roadmap for Semiconductors, as outlined in the introductory chapter of this thesis. Until then only one thing is sure, there will always be something left to learn about diffusion barrier layers. 


\section{BIBLIOGRAPHY}

[1] E. Suhir, Microelectronics and photonics - the future, Microelectr. J. 31 (2000) 839-851.

[2] International Technology Roadmap for Semiconductors, (2013). http://www.itrs.net/home.html (accessed February 4, 2015).

[3] A.E. Kaloyeros, E. Eisenbraun, Ultrathin diffusion barriers/liners for gigascale copper metallization, Annu. Rev. Mater. Sci. 30 (2000) 363-385.

[4] C. Lee, Y.-L. Kuo, The evolution of diffusion barriers in copper metallization, JOM. 59 (2007) 44-49.

[5] F. Magnus, A.S. Ingason, S. Olafsson, J.T. Gudmundsson, Nucleation and resistivity of ultrathin TiN films grown by high-power impulse magnetron sputtering, IEEE Electron Device Lett. 33 (2012) 1045-1047.

[6] A. Gupta, H. Wang, A. Kvit, G. Duscher, J. Narayan, Effect of microstructure on diffusion of copper in TiN films, J. Appl. Phys. 93 (2003) 5210-5214.

[7] M. Moriyama, T. Kawazoe, M. Tanaka, M. Murakami, Correlation between microstructure and barrier properties of TiN thin films used $\mathrm{Cu}$ interconnects, Thin Solid Films. 416 (2002) 136-144.

[8] A.K. Petford-Long, A.N. Chiaramonti, Transmission electron microscopy of multilayer thin films, Annu. Rev. Mater. Res. 38 (2008) 559-584.

[9] M. Mühlbacher, F. Mendez-Martin, B. Sartory, N. Schalk, J. Keckes, J. Lu, L. Hultman, C. Mitterer, Copper diffusion into single-crystalline TiN studied by transmission electron microscopy and atom probe tomography, Thin Solid Films. 574 (2015) 103-109.

[10] Encyclopædia Britannica Online, s. v. "diffusion," (2015). http://global.britannica.com/EBchecked/topic/163068/diffusion (accessed June 10, 2015).

[11] M. Reibold, P. Paufler, A.A. Levin, W. Kochmann, N. Pätzke, D.C. Meyer, Materials: Carbon nanotubes in an ancient Damascus sabre, Nature. 444 (2006) 286.

[12] J. Dalton, A new system of chemical philosophy. Part I., R. Bickerstaff, London, 1808.

[13] T. Graham, On the law of the diffusion of gases, Philos. Mag. 2 (1833) 175-190.

[14] H. Mehrer, N.A. Stolwijk, Heroes and highlights in the history of diffusion, Diffus. Fundam. 11 (2009) 1-32.

[15] A. Fick, Ueber Diffusion, Ann. Phys. 170 (1855) 59-86. 
[16] D. Gupta, Diffusion in bulk solids and thin films: Some phenomenological examples, in: D. Gupta (Ed.), Diffusion Processes in Advanced Technological Materials, 1st ed., William Andrew Publishing, Norwich, NY, 2005: pp. 1-68.

[17] M. Ohring, Materials Sscience of thin films: Deposition and structure, 2nd ed., Academic Press, San Diego, 2002.

[18] H. Mehrer, Diffusion in solids, 1st ed., Springer, Berlin-Heidelberg-New York, 2007.

[19] D.S. Wilkinson, Mass transport in solids and fluids, Cambridge University Press, Cambridge, 2000.

[20] T.L. Chow, Mathematical methods for physicists, Cambridge University Press, Cambridge, 2000.

[21] A. Einstein, Investigations on the theory of the Brownian movement, Dover Publications, Inc., Mineola, NY, 1956.

[22] S.R.L. Couling, R. Smoluchowski, Anisotropy of diffusion in grain boundaries, J. Appl. Phys. 25 (1954) 1538-1542.

[23] D. Turnbull, R.E. Hoffman, The effect of relative crystal and boundary orientations on grain boundary diffusion rates, Acta Metall. 2 (1954) 419426.

[24] L.G. Harrison, Influence of dislocations on diffusion kinetics in solids with particular reference to the alkali halides, Trans. Faraday Soc. 57 (1961) 1191-1199.

[25] R.T.P. Whipple, Concentration contours in grain boundary diffusion, Philos. Mag. 45 (1954) 1225-1236.

[26] T. Suzuoka, Lattice and grain boundary diffusion in polycrystals, Trans. Japan Inst. Met. 2 (1961) 25-33.

[27] J. Sommer, C. Herzig, Direct determination of grain-boundary and dislocation self-diffusion coefficients in silver from experiments in type-C kinetics, J. Appl. Phys. 72 (1992) 2758-2766.

[28] P. Gas, D.L. Beke, J. Bernardino, Grain-boundary diffusion: Analysis of the C kinetic regime, Philos. Mag. Lett. 65 (1992) 133-139.

[29] S.I. Shah, G.H. Jaffari, E. Yassitepe, B. Ali, Evaporation: Processes, bulk microstructures, and mechanical properties, in: Peter M. Martin (Ed.), Handbook of Deposition Technologies for Films and Coatings, 3rd ed., William Andrew Publishing, Oxford, 2010: pp. 135-252.

[30] D.M. Mattox, Handbook of physical vapor deposition (PVD) processing, 1st ed., Noyes Publications, Westwood, NJ, 1998.

[31] D. Depla, S. Mahieu, J.E. Greene, Sputter deposition processes, in: P.M. Martin (Ed.), Handbook of Deposition Technologies for Films and Coatings, 3rd ed., William Andrew Publishing, Oxford, 2010: pp. 253296.

[32] G. Bräuer, B. Szyszka, M. Vergöhl, R. Bandorf, Magnetron sputtering Milestones of 30 years, Vacuum. 84 (2010) 1354-1359. 
[33] P.J. Kelly, R.D. Arnell, Magnetron sputtering: a review of recent developments and applications, Vacuum. 56 (2000) 159-172.

[34] P.H. Mayrhofer, F. Kunc, J. Musil, C. Mitterer, A comparative study on reactive and non-reactive unbalanced magnetron sputter deposition of TiN coatings, Thin Solid Films. 415 (2002) 151-159.

[35] W.D. Sproul, D.J. Christie, D.C. Carter, Control of reactive sputtering processes, Thin Solid Films. 491 (2005) 1-17.

[36] J.E. Greene, Thin film nucleation, growth, and microstructural evolution: An atomic scale view, in: P.M. Martin (Ed.), Handbook of Deposition Technologies for Films and Coatings, 3rd ed., William Andrew Publishing, Oxford, 2010: pp. 554-620.

[37] Z. Gai, J.Y. Howe, J. Guo, D.A. Blom, E.W. Plummer, J. Shen, Selfassembled FePt nanodot arrays with mono-dispersion and -orientation, Appl. Phys. Lett. 86 (2005) 0231071-0231073.

[38] U. Diebold, J.-M. Pan, T.E. Madey, Growth mode of ultrathin copper overlayers on $\mathrm{TiO}_{2}$ (110), Phys. Rev. B. 47 (1993) 3868-3876.

[39] C. Friesen, C. V Thompson, Reversible stress relaxation during precoalescence interruptions of Volmer-Weber thin film growth, Phys. Rev. Lett. 89 (2002) 1261031-1261034.

[40] J.A. Venables, Atomic processes in crystal growth, Surf. Sci. 299-300 (1994) 798-817.

[41] R.S. Wagner, Adsorption and growth of cadmium on polycrystalline tungsten, J. Appl. Phys. 42 (1971) 3948-3959.

[42] N.-E. Lee, D.G. Cahill, J.E. Greene, Evolution of surface roughness in epitaxial $\mathrm{Si}_{0.7} \mathrm{Ge}_{0.3}(001)$ as a function of growth temperature $\left(200-600{ }^{\circ} \mathrm{C}\right)$ and $\operatorname{Si}(001)$ substrate miscut, J. Appl. Phys. 80 (1996) 2199-2210.

[43] J. Knall, S.A. Barnett, J.-E. Sundgren, J.E. Greene, Adsorption and desorption kinetics of In on Si(100), Surf. Sci. 209 (1989) 314-334.

[44] C. Eisenmenger-Sittner, H. Bangert, H. Störi, J. Brenner, P.B. Barna, Stranski-Krastanov growth of $\mathrm{Sn}$ on a polycrystalline Al film surface initiated by the wetting of Al by Sn, Surf. Sci. 489 (2001) 161-168.

[45] J. Bøttiger, J. Chevallier, J.H. Petersen, N. Schell, W. Matz, A. Mücklich, Observation of the growth mode of TiN during magnetron sputtering using synchrotron radiation, J. Appl. Phys. 91 (2002) 5429-5433.

[46] P. Patsalas, S. Logothetidis, Interface properties and structural evolution of TiN/Si and TiN/GaN heterostructures, J. Appl. Phys. 93 (2003) 989-998.

[47] E.G. Bauer, B.W. Dodson, D.J. Ehrlich, L.C. Feldman, C.P. Flynn, M.W. Geis, J.P. Harbison, R.J. Matyi, P.S. Peercy, P.M. Petroff, J.M. Phillips, G.B. Stringfellow, A. Zangwill, Fundamental issues in heteroepitaxy-A Department of Energy, Council on Materials Science Panel Report, J. Mater. Res. 5 (1990) 852-894. 
[48] G.G. Jernigan, P.E. Thompson, Temperature dependence of atomic scale morphology in Si homoepitaxy between 350 and $800{ }^{\circ} \mathrm{C}$ on $\mathrm{Si}(100)$ by molecular beam epitaxy, J. Vac. Sci. Technol. A . 19 (2001) 2307-2311.

[49] K. Kutschej, B. Rashkova, J. Shen, D. Edwards, C. Mitterer, G. Dehm, Experimental studies on epitaxially grown TiN and VN films, Thin Solid Films. 516 (2007) 369-373.

[50] L. Hultman, J.-E. Sundgren, L.C. Markert, J.E. Greene, Ar and excess N incorporation in epitaxial TiN films grown by reactive bias sputtering in mixed Ar/N2 and pure N2 discharges, J. Vac. Sci. Technol. A . 7 (1989) 1187-1193.

[51] H. Ljungcrantz, M. Odén, L. Hultman, J.E. Greene, J.-E. Sundgren, Nanoindentation studies of single-crystal (001)-, (011)-, and (111)-oriented TiN layers on MgO, J. Appl. Phys. 80 (1996) 6725-6733.

[52] B.W. Karr, I. Petrov, D.G. Cahill, J.E. Greene, Morphology of epitaxial TiN(001) grown by magnetron sputtering, Appl. Phys. Lett. 70 (1997) 1703-1705.

[53] A.M. Minor, E.A. Stach, J.W. Morris, I. Petrov, In-situ nanoindentation of epitaxial TiN/MgO (001) in a transmission electron microscope, J. Electron. Mater. 32 (2003) 1023-1027.

[54] L. Hultman, S.A. Barnett, J.-E. Sundgren, J.E. Greene, Growth of epitaxial TiN films deposited on $\mathrm{MgO}(100)$ by reactive magnetron sputtering: The role of low-energy ion irradiation during deposition, J. Cryst. Growth. 92 (1988) 639-656.

[55] B.O. Johansson, J.E. Sundgren, J.E. Greene, A. Rocket, S.A. Barnett, Growth and properties of single crystal TiN films deposited by reactive magnetron sputtering, J. Vac. Sci. Technol. A . 3 (1985) 303-307.

[56] International Center for Diffraction Data, Powder Diffraction File 00-0450946, PDF-2/Release, 2007.

[57] International Center for Diffraction Data, Powder Diffraction File 00-0381420, PDF-2/Release, 2007.

[58] International Center for Diffraction Data, Powder Diffraction File 00-0040836, PDF-2/Release, 2007.

[59] S. Cazottes, Z.L. Zhang, R. Daniel, J.S. Chawla, D. Gall, G. Dehm, Structural characterization of a $\mathrm{Cu} / \mathrm{MgO}(001)$ interface using CS-corrected HRTEM, Thin Solid Films. 519 (2010) 1662-1667.

[60] Z. Zhang, Y. Long, S. Cazottes, R. Daniel, C. Mitterer, G. Dehm, The peculiarity of the metal-ceramic interface, Sci. Rep. 5 (2015) 11460.

[61] G. Abadias, Y.Y. Tse, Determination of intrinsic stresses in textured and epitaxial TiN thin films deposited by dual ion beam sputtering, Surf. Coat. Tech. 180-181 (2004) 33-40.

[62] P.B. Barna, M. Adamik, Fundamental structure forming phenomena of polycrystalline films and the structure zone models, Thin Solid Films. 317 (1998) 27-33. 
[63] C. V Thompson, Grain growth in thin films, Annu. Rev. Mater. Sci. 20 (1990) 245-268.

[64] J.A. Thornton, High rate thick film growth, Annu. Rev. Mater. Sci. 7 (1977) 239-260.

[65] A. Anders, A structure zone diagram including plasma-based deposition and ion etching, Thin Solid Films. 518 (2010) 4087-4090.

[66] D.M. Mattox, Particle bombardment effects on thin-film deposition: A review, J. Vac. Sci. Technol. A .7 (1989) 1105-1114.

[67] S. Grasser, Influence of pulsed bias on $\mathrm{CrN}$ coatings prepared by reactive magnetron sputtering, Diploma thesis, Montanuniversität Leoben, 2008.

[68] J.-E. Sundgren, B.-O. Johansson, H.T.G. Hentzell, S.-E. Karlsson, Mechanisms of reactive sputtering of titanium nitride and titanium carbide III: Influence of substrate bias on composition and structure, Thin Solid Films. 105 (1983) 385-393.

[69] I. Petrov, L. Hultman, J.-E. Sundgren, J.E. Greene, Polycrystalline TiN films deposited by reactive bias magnetron sputtering: Effects of ion bombardment on resputtering rates, film composition, and microstructure, J. Vac. Sci. Technol. A . 10 (1992) 265-272.

[70] P. Patsalas, C. Charitidis, S. Logothetidis, The effect of substrate temperature and biasing on the mechanical properties and structure of sputtered titanium nitride thin films, Surf. Coat. Tech. 125 (2000) 335-340.

[71] R. Daniel, private communication, 2012.

[72] L.E. Toth, Transition metal carbides and nitrides, Academic Press, New York, 1971.

[73] H. Okamoto, N-Ti (Nitrogen-Titanium), J. Phase Equilibria Diffus. 34 (2012) 151-152.

[74] J.-E. Sundgren, Structure and properties of TiN coatings, Thin Solid Films. 128 (1985) 21-44.

[75] J.-E. Sundgren, B.-O. Johansson, S.-E. Karlsson, H.T.G. Hentzell, Mechanisms of reactive sputtering of titanium nitride and titanium carbide II: Morphology and structure, Thin Solid Films. 105 (1983) 367-384.

[76] J.-E. Sundgren, B.-O. Johansson, S.-E. Karlsson, Mechanisms of reactive sputtering of titanium nitride and titanium carbide I: Influence of process parameters on film composition, Thin Solid Films. 105 (1983) 353-366.

[77] R.D. Vispute, R. Chowdhury, P. Tiwari, J. Narayan, Pulsed laser deposition and characterization of epitaxial $\mathrm{Cu} / \mathrm{TiN} / \mathrm{Si}(100)$ heterostructures, Appl. Phys. Lett. 65 (1994) 2565-2567.

[78] J. Narayan, P. Tiwari, X. Chen, J. Singh, R. Chowdhury, T. Zheleva, Epitaxial growth of TiN films on (100) silicon substrates by laser physical vapor deposition, Appl. Phys. Lett. 61 (1992) 1290-1292.

[79] R.D. Vispute, J. Narayan, Epitaxial TiN based contacts for silicon devices, J. Electron. Mater. 25 (1996) 1740-1747. 
[80] P. Patsalas, C. Charitidis, S. Logothetidis, The effect of substrate temperature and biasing on the mechanical properties and structure of sputtered titanium nitride thin films, Surf. Coat. Tech. 125 (2000) 335-340.

[81] O.R. Shojaei, A. Karimi, Comparison of mechanical properties of TiN thin films using nanoindentation and bulge test, Thin Solid Films. 332 (1998) 202-208.

[82] C. Stampfl, W. Mannstadt, R. Asahi, A. Freeman, Electronic structure and physical properties of early transition metal mononitrides: Densityfunctional theory LDA, GGA, and screened-exchange LDA FLAPW calculations, Phys. Rev. B. 63 (2001) 1-11.

[83] R.S. Ningthoujam, N.S. Gajbhiye, Synthesis, electron transport properties of transition metal nitrides and applications, Prog. Mater. Sci. 70 (2015) 50-154.

[84] W. Tsai, M. Delfino, J.A. Fair, D. Hodul, Temperature dependence of the electrical resistivity of reactively sputtered TiN films, J. Appl. Phys. 73 (1993) 4462-4467.

[85] N. Savvides, B. Window, Electrical transport, optical properties, and structure of TiN films synthesized by low-energy ion assisted deposition, J. Appl. Phys. 64 (1988) 225-234.

[86] M. Kawamura, Y. Abe, H. Yanagisawa, K. Sasaki, Characterization of TiN films prepared by a conventional magnetron sputtering system: Influence of nitrogen flow percentage and electrical properties, Thin Solid Films. 287 (1996) 115-119.

[87] S. Niyomsoan, W. Grant, D.L. Olson, B. Mishra, Variation of color in titanium and zirconium nitride decorative thin films, Thin Solid Films. 415 (2002) 187-194.

[88] A. Mumtaz, W.H. Class, Color of titanium nitride prepared by reactive dc magnetron sputtering, J. Vac. Sci. Technol. 20 (1982) 345-348.

[89] S.P. Murarka, Diffusion barriers in semiconductor devices/circuits, in: D. Gupta (Ed.), Diffusion Processes in Advanced Technological Materials, 1st ed., William Andrew Publishing, Norwich, NY, 2005: pp. 239-281.

[90] M.-A. Nicolet, Diffusion barriers in layered contact structures, J. Vac. Sci. Technol. 19 (1981) 786-793.

[91] M.-A. Nicolet, Diffusion barriers in thin films, Thin Solid Films. 52 (1978) 415-443.

[92] K. Maex, M.R. Baklanov, D. Shamiryan, F. Lacopi, S.H. Brongersma, Z.S. Yanovitskaya, Low dielectric constant materials for microelectronics, J. Appl. Phys. 93 (2003) 8793-8841.

[93] C.-K. Hu, J.M.E. Harper, Copper interconnections and reliability, Mater. Chem. Phys. 52 (1998) 5-16.

[94] M.B. Chamberlain, Diffusion of copper in thin TiN films, Thin Solid Films. 91 (1982) 155-162. 
[95] M. Mändl, H. Hoffmann, P. Kücher, Diffusion barrier properties of Ti/TiN investigated by transmission electron microscopy, J. Appl. Phys. 68 (1990) 2127-2132.

[96] W. Sinke, G.P.A. Frijlink, F.W. Saris, Oxygen in titanium nitride diffusion barriers, Appl. Phys. Lett. 47 (1985) 471-473.

[97] J.O. Olowolafe, J. Li, J.W. Mayer, E.G. Colgan, Effects of oxygen in $\mathrm{TiN}_{\mathrm{x}}$ on the diffusion of $\mathrm{Cu}$ in $\mathrm{Cu} / \mathrm{TiN} / \mathrm{Al}$ and $\mathrm{Cu} / \mathrm{TiN}_{\mathrm{x}} / \mathrm{Si}$ structures, Appl. Phys. Lett. 58 (1991) 469-471.

[98] K.T. Nam, A. Datta, S.-H. Kim, K.-B. Kim, Improved diffusion barrier by stuffing the grain boundaries of TiN with a thin $\mathrm{Al}$ interlayer for $\mathrm{Cu}$ metallization, Appl. Phys. Lett. 79 (2001) 2549-2551.

[99] K.-C. Park, K.-B. Kim, Effect of annealing of titanium nitride on the diffusion barrier property in $\mathrm{Cu}$ metallization, J. Electrochem. Soc. 142 (1995) 3109-3115.

[100] W.-H. Lee, Y.-L. Kuo, H.-J. Huang, C. Lee, Effect of density on the diffusion barrier property of $\mathrm{TiN}_{\mathrm{x}}$ films between $\mathrm{Cu}$ and $\mathrm{Si}$, Mater. Chem. Phys. 85 (2004) 444-449.

[101] F. Krumeich, Properties of electrons, their interactions with matter and applications in electron microscopy, (2015). http:/www.microscopy.ethz.ch/downloads/Interactions.pdf (accessed January 25, 2015).

[102] D.B. Williams, C.B. Carter, Transmission electron microscopy: A textbook for materials science, Springer Science+Business Media, New York, 2009.

[103] J.M. LeBeau, S.D. Findlay, L.J. Allen, S. Stemmer, Quantitative atomic resolution scanning transmission electron microscopy, Phys. Rev. Lett. 100 (2008) 2061011-2061014.

[104] T.F. Kelly, M.K. Miller, K. Rajan, S.P. Ringer, Atomic-scale tomography: A 2020 vision, Microsc. Microanal. 19 (2013) 652-664.

[105] R.H. Wade, A brief look at imaging and contrast transfer, Ultramicroscopy. 46 (1992) 145-156.

[106] J. Palisaitis, Electron energy loss spectroscopy of III-nitride semiconductors, Licentiate thesis, Linköping University, 2011.

[107] O. Scherzer, The theoretical resolution limit of the electron microscope, J. Appl. Phys. 20 (1949) 20-29.

[108] B. Fultz, J. Howe, Transmission electron microscopy and diffractometry of materials, 4th ed., Springer, Berlin-Heidelberg-New York, 2013.

[109] P.J. Goodhew, J. Humphreys, R. Beanland, Electron microscopy and analysis, 3rd ed., Taylor \& Francis, London, 2001.

[110] W. Sigle, Analytical transmission electron microscopy, Annu. Rev. Mater. Res. 35 (2005) 239-314.

[111] D.J. Larson, T.J. Prosa, R.M. Ulfig, B.P. Geiser, T.F. Kelly, Local electrode atom probe tomography, Springer Science+Business Media, New York, 2013. 
[112] B. Gault, M.P. Moody, J.M. Cairney, S.P. Ringer, Atom probe microscopy, Springer Science+Business Media, New York, 2012.

[113] H. Gnaser, Atom probe tomography of nanostructures, Surf. Interface Anal. 46 (2014) 383-388.

[114] T.F. Kelly, D.J. Larson, Atom probe tomography 2012, Annu. Rev. Mater. Res. 42 (2012) 1-31.

[115] D.N. Seidman, Three-dimensional atom-probe tomography: Advances and applications, Annu. Rev. Mater. Res. 37 (2007) 127-158.

[116] A. Cerezo, G.D.W. Smith, P.H. Clifton, Measurement of temperature rises in the femtosecond laser pulsed three-dimensional atom probe, Appl. Phys. Lett. 88 (2006) 1541031-1541033.

[117] B. Gault, F. Vurpillot, A. Bostel, A. Menand, B. Deconihout, Estimation of the tip field enhancement on a field emitter under laser illumination, Appl. Phys. Lett. 86 (2005) 0941011-0941013.

[118] D. Larson, B. Wissman, R. Martens, R. Viellieux, T. Kelly, T. Gribb, H. Erskine, N. Tabat, Advances in atom probe specimen fabrication from planar multilayer thin film structures, Microsc. Microanal. 7 (2001) 24-31.

[119] E.A. Marquis, N.A. Yahya, D.J. Larson, M.K. Miller, R.I. Todd, Probing the improbable: Imaging $\mathrm{C}$ atoms in alumina, Mater. Today. 13 (2010) 3436.

[120] G. Sha, S.P. Ringer, Effect of laser pulsing on the composition measurement of an $\mathrm{Al}-\mathrm{Mg}-\mathrm{Si}-\mathrm{Cu}$ alloy using three-dimensional atom probe, Ultramicroscopy. 109 (2009) 580-584.

[121] P. Bas, A. Bostel, B. Deconihout, D. Blavette, A general protocol for the reconstruction of 3D atom probe data, Appl. Surf. Sci. 87-88 (1995) 298304.

[122] B. Geiser, D. Larson, E. Oltman, S. Gerstl, D. Reinhard, T. Kelly, T. Prosa, Wide-field-of-view atom probe reconstruction, Microsc. Microanal. 15 (2009) 292-293.

[123] L.J.S. Johnson, M. Thuvander, K. Stiller, M. Odén, L. Hultman, Blind deconvolution of time-of-flight mass spectra from atom probe tomography, Ultramicroscopy. 132 (2013) 60-64.

[124] D.J. Larson, T.J. Prosa, B.P. Geiser, W.F. Egelhoff, Effect of analysis direction on the measurement of interfacial mixing in thin metal layers with atom probe tomography, Ultramicroscopy. 111 (2011) 506-511.

[125] E.A. Marquis, B.P. Geiser, T.J. Prosa, D.J. Larson, Evolution of tip shape during field evaporation of complex multilayer structures, J. Microsc. 241 (2011) 225-33.

[126] D.J. Larson, B.P. Geiser, T.J. Prosa, S.S.A. Gerstl, D.A. Reinhard, T.F. Kelly, Improvements in planar feature reconstructions in atom probe tomography, J. Microsc. 243 (2011) 15-30. 
[127] L.J.S. Johnson, M. Thuvander, K. Stiller, M. Odén, L. Hultman, Spinodal decomposition of $\mathrm{Ti}_{0.33} \mathrm{Al}_{0.67} \mathrm{~N}$ thin films studied by atom probe tomography, Thin Solid Films. 520 (2012) 4362-4368.

[128] R. Rachbauer, S. Massl, E. Stergar, D. Holec, D. Kiener, J. Keckes, J. Patscheider, M. Stiefel, H. Leitner, P.H. Mayrhofer, Decomposition pathways in age hardening of Ti-Al-N films, J. Appl. Phys. 110 (2011) 0235151-02351510.

[129] R. Rachbauer, E. Stergar, S. Massl, M. Moser, P.H. Mayrhofer, Threedimensional atom probe investigations of $\mathrm{Ti}-\mathrm{Al}-\mathrm{N}$ thin films, Scr. Mater. 61 (2009) 725-728.

[130] T.F. Kelly, D.J. Larson, K. Thompson, R.L. Alvis, J.H. Bunton, J.D. Olson, B.P. Gorman, Atom probe tomography of electronic materials, Annu. Rev. Mater. Res. 37 (2007) 681-727.

[131] G. Greczynski, J. Lu, S. Bolz, W. Kölker, C. Schiffers, O. Lemmer, I. Petrov, J.E. Greene, L. Hultman, Novel strategy for low-temperature, highrate growth of dense, hard, and stress-free refractory ceramic thin films, J. Vac. Sci. Technol. A . 32 (2014) 0415151-04151512. 



\section{PUBLICATIONS}

\section{List of included publications:}

Paper I. Copper diffusion into single-crystalline TiN studied by transmission electron microscopy and atom probe tomography.

M. Mühlbacher, F. Mendez-Martin, B. Sartory, N. Schalk, J. Keckes, J. Lu, L. Hultman, and C. Mitterer.

Thin Solid Films. 574 (2015) 103-109.

Paper II. Cu diffusion in single-crystal and polycrystalline TiN barrier layers: A high-resolution experimental study supported by first-principles calculations.

M. Mühlbacher, A. S. Bochkarev, F. Mendez-Martin, B. Sartory, L. Chitu, M. N. Popov, P. Puschnig, J. Spitaler, H. Ding, N. Schalk, J. Lu, L. Hultman, and C. Mitterer.

Submitted for publication.

\section{Author's contributions (supervision not included):}

Paper I: I planned and performed the depositions and annealing treatments, carried out the APT and TEM investigations, and evaluated and interpreted all the data. I wrote the manuscript.

Paper II: I planned and performed the depositions and annealing treatments, carried out the XRD, APT, and TEM investigations, and evaluated and interpreted all the data. With the exception of the theoretical sections (section IV and parts of section II), I wrote the manuscript.

Related publications not included in this thesis:

- Functional thin films for display and microelectronics applications. C. Mitterer, T. Jörg, R. Franz, M. Mühlbacher, B. Sartory, F. Mendez-Martin, and N. Schalk, BHM Berg- und Hüttenmännische Monatshefte. 160 (2015) 231-234.

- $\mathrm{TiO}_{2}(100) / \mathrm{Al}_{2} \mathrm{O}_{3}(0001)$ interface: $\mathrm{A}$ first-principles study supported by experiment. M.N. Popov, J. Spitaler, M. Mühlbacher, C. Walter, J. Keckes, C. Mitterer, and C. Draxl, Phys. Rev. B. 86 (2012) 205309. 
